\title{
Global Existence for Two Singular One-Dimensional Nonlinear Viscoelastic Equations with respect to Distributed Delay Term
}

\author{
Abdelbaki Choucha, ${ }^{1}$ Salah Mahmoud Boulaaras $\mathbb{D}^{2,3}$ Djamel Ouchenane, ${ }^{4}$ \\ and Ali Allahem ${ }^{5}{ }^{5}$ \\ ${ }^{1}$ Laboratory of Operator Theory and PDEs: Foundations and Applications, Department of Mathematics, Faculty of Exact Sciences, \\ University of El Oued, El Oued, Algeria \\ ${ }^{2}$ Department of Mathematics, College of Sciences and Arts, ArRass, Qassim University, Saudi Arabia \\ ${ }^{3}$ Laboratory of Fundamental and Applied Mathematics of Oran (LMFAO), University of Oran 1, Ahmed Benbella, Algeria \\ ${ }^{4}$ Laboratory of Pure and Applied Mathematics, Amar Telidji Laghouat University, Algeria \\ ${ }^{5}$ Department of Mathematics, College of Sciences, Qassim University, Saudi Arabia
}

Correspondence should be addressed to Salah Mahmoud Boulaaras; s.boularas@qu.edu.sa and Ali Allahem; aallahem@qu.edu.sa

Received 31 December 2020; Revised 24 January 2021; Accepted 2 February 2021; Published 16 February 2021

Academic Editor: Mahmoud A. Zaky

Copyright (c) 2021 Abdelbaki Choucha et al. This is an open access article distributed under the Creative Commons Attribution License, which permits unrestricted use, distribution, and reproduction in any medium, provided the original work is properly cited.

In this current work, we are interested in a system of two singular one-dimensional nonlinear equations with a viscoelastic, general source and distributed delay terms. The existence of a global solution is established by the theory of potential well, and by using the energy method with the function of Lyapunov, we prove the general decay result of our system.

\section{Introduction}

We are interested in the following system:

$$
\left\{\begin{array}{l}
u_{t t}-\frac{1}{x}\left(x u_{x}\right)_{x}+\int_{0}^{t} g_{1}(t-s) \frac{1}{x}\left(x u_{x}(x, s)\right)_{x} d s+\mu_{1} u_{t} \\
+\int_{\tau_{1}}^{\tau_{2}}\left|\mu_{2}(\rho)\right| u_{t}(x, t-\rho) d \rho=f_{1}(u, v), \text { in } Q, \\
v_{t t}-\frac{1}{x}\left(x v_{x}\right)_{x}+\int_{0}^{t} g_{2}(t-s) \frac{1}{x}\left(x v_{x}(x, s)\right)_{x} d s+\mu_{3} v_{t} \\
+\int_{\tau_{1}}^{\tau_{2}}\left|\mu_{4}(\rho)\right| v_{t}(x, t-\rho) d \rho=f_{2}(u, v), \text { in } Q,
\end{array}\right.
$$

with

$$
\left\{\begin{array}{l}
u(x, 0)=u_{0}(x), u_{t}(x, 0)=u_{1}(x), x \in(0, L), \\
v(x, 0)=v_{0}(x), v_{t}(x, 0)=v_{1}(x), x \in(0, L), \\
u_{t}(x,-t)=f_{0}(x, t), v_{t}(x,-t)=g_{0}(x, t), t \in\left(0, \tau_{2}\right), \\
u(L, t)=v(L, t)=0, \int_{0}^{L} x u(x, t) d x=\int_{0}^{L} x v(x, t) d x=0,
\end{array}\right.
$$

where $Q=(0, L) \times(0, T), L<\infty, T<\infty, g_{1}(),. g_{2}():. \mathbb{R}^{+}$ $\longrightarrow \mathbb{R}^{+}, \mu_{1}, \mu_{3}>0$, the second integral represents the distributed delay and $\mu_{2}, \mu_{4}:\left[\tau_{1}, \tau_{2}\right] \longrightarrow \mathbb{R}$ are bounded functions, where $\tau_{1}, \tau_{2}$ are two real numbers satisfying 0 $\leq \tau_{1}<\tau_{2}$, and $f_{1}(.,),. f_{2}(.,):. \mathbb{R}^{2} \longrightarrow \mathbb{R}$ are defined functions later.

Three decades ago, these problems that arise in onedimensional elasticity have been studied and developed with regard to viscosity with long-term memory. And it has been studied in many fields of science, engineering, medical sciences, and chemistry, as well as population and other matters; see, for example, [1-24]. Recently, in the absence of delay $\left(\mu_{i}=0, i=1 . .4\right)$, problem (1) was studied in [25], and also later in [26], the authors considered problem (1) with localized frictional damping term. We also know that delay, especially distributed delay, is a phenomenon in our life and is almost found in various fields, and its inclusion in any problem makes it more important. The distributed delay in many works has been studied and many authors have taken care of it, for example, [5, 9, 27, 28]. Based on all this and the results of the research papers $[14,15,17$, $28-30,31]$, the introduction of the term distributed delay as 
a damping mechanism in problem (1) makes it a new problem from what has been previously studied.

And we have divided this paper into the following. We present in the second section the definitions, basics, and theories of function spaces that are required throughout the rest of the paper. In Section 3, we present the energy function while proving to be decreasing. And in the final section, the general decay is obtained by applying the energy method and the function of Lyapunov.

\section{Preliminaries}

Let $L_{x}^{p}=L_{x}^{p}((0, L))$ be the weighted Banach space equipped with the norm

$$
\|u\|_{L_{x}^{p}}=\left(\int_{0}^{L} x|u|^{p} d x\right)^{1 / p}
$$

$H=L_{x}^{2}((0, L))$ be the Hilbert space of square integral functions having the finite norm

$$
\|u\|_{H}=\left(\int_{0}^{L} x u^{2} d x\right)^{1 / 2}
$$

and $K=L_{x}^{2}\left((0, L) \times(0,1) \times\left(\tau_{1}, \tau_{2}\right)\right)$ be the Hilbert space equipped with the norm

$$
\|z\|_{K, \mu_{2}}=\int_{0}^{1} \int_{\tau_{1}}^{\tau_{2}}\left|\mu_{2}(\rho)\right|\|z\|_{H} d \rho d \rho .
$$

$V=V_{x}^{1}$ is the Hilbert space equipped with the norm

$$
\begin{aligned}
\|u\|_{V} & =\left(\|u\|_{H}^{2}+\left\|u_{x}\right\|_{H}^{2}\right)^{1 / 2}, \\
V_{0} & =\{u \in V \text { such that } u(L)=0\} .
\end{aligned}
$$

Theorem 1 [27]. For $2<p<4$ and $\forall v$ in $V_{0}$, we have

$$
\int_{0}^{L} x|v|^{p} d x \leq C_{*}\left\|v_{x}\right\|_{H=L_{x}^{2}(0, L)}^{p},
$$

where $C_{*}$ is a constant depending on $L$ and $p$ only.

As in [18], introducing the new variables

$$
\left\{\begin{array}{l}
z(x, \rho, \varrho, t)=u_{t}(x, t-\varrho \rho) \\
y(x, \rho, \varrho, t)=v_{t}(x, t-\varrho \rho)
\end{array}\right.
$$

yields

$$
\begin{aligned}
& \left\{\begin{array}{l}
\mathrm{\varrho} z_{t}(x, \rho, \varrho, t)+z_{\rho}(x, \rho, \varrho, t)=0, \\
z(x, 0, \varrho, t)=u_{t}(x, t),
\end{array}\right. \\
& \left\{\begin{array}{l}
\mathrm{\varrho} y_{t}(x, \rho, \varrho, t)+y_{\rho}(x, \rho, \varrho, t)=0, \\
y(x, 0, \varrho, t)=v_{t}(x, t) .
\end{array}\right.
\end{aligned}
$$

Problem (1) arrives at

$$
\left\{\begin{array}{l}
u_{t t}-\frac{1}{x}\left(x u_{x}\right)_{x}+\int_{0}^{t} g_{1}(t-s) \frac{1}{x}\left(x u_{x}(x, s)\right)_{x} d s+\mu_{1} u_{t} \\
+\int_{\tau_{1}}^{\tau_{2}}\left|\mu_{2}(\varrho)\right| u_{t}(x, t-\varrho) d \varrho=f_{1}(u, v), \\
v_{t t}-\frac{1}{x}\left(x v_{x}\right)_{x}+\int_{0}^{t} g_{2}(t-s) \frac{1}{x}\left(x v_{x}(x, s)\right)_{x} d s+\mu_{3} v_{t} \\
+\int_{\tau_{1}}^{\tau_{2}}\left|\mu_{4}(\varrho)\right| v_{t}(x, t-\varrho) d \varrho=f_{2}(u, v), \\
\varrho z_{t}(x, \rho, \varrho, t)+z_{\rho}(x, \rho, \varrho, t)=0 \\
\varrho y_{t}(x, \rho, \varrho, t)+y_{\rho}(x, \rho, \varrho, t)=0
\end{array}\right.
$$

where

$$
(x, \rho, s, t) \in(0, L) \times(0,1) \times\left(\tau_{1}, \tau_{2}\right) \times(0, \infty) .
$$

With the initial data and boundary conditions

$\left\{\begin{array}{l}(u(x, 0), v(x, 0))=\left(u_{0}(x), v_{0}(x)\right), \text { in }(0, L), \\ \left(u_{t}(x, 0), v_{t}(x, 0)\right)=\left(u_{1}(x), v_{1}(x)\right), \text { in }(0, L), \\ \left(u_{t}(x,-t), v_{t}(x,-t)\right)=\left(f_{0}(x, t), g_{0}(x, t)\right), \text { in }(0, L) \times\left(0, \tau_{2}\right), \\ u(0, t)=u(L, t)=v(0, t)=v(L, t)=0, \\ z(x, \rho, \varrho, 0)=f_{0}(x, \rho \varrho), \text { in }(0, L) \times(0,1) \times\left(0, \tau_{2}\right), \\ y(x, \rho, \varrho, 0)=g_{0}(x, \rho \varrho), \\ u(L, t)=v(L, t)=0, \int_{0}^{L} x u(x, t) d x=\int_{0}^{L} x v(x, t) d x=0 .\end{array}\right.$

We have the following assumptions:

(G1) $g_{i}(t): \mathbb{R}^{+} \longrightarrow \mathbb{R}^{+}$are $C^{1}$, nonincreasing functions satisfying

$$
\left\{\begin{array}{l}
g_{i}(s) \geq 0, \quad g_{i}^{\prime}(s) \leq 0, \\
g_{i}(0)>0,1-\int_{0}^{\infty} g_{i}(s) d s=l_{i}>0, i=1,2,
\end{array}\right.
$$

(G2) $\exists \xi(t)>0$ a differentiable function, such that

$$
g_{i}^{\prime}(t) \leq-\xi(t) g_{i}^{\sigma}(t), i=1,2, t \geq 0,1 \leq \sigma<\frac{3}{2},
$$

and $\xi(t)$ satisfies for some $l<1$

$$
\xi^{\prime}(t) \leq 0,\left|\frac{\xi^{\prime}(t)}{\xi(t)}\right| \leq l, \int_{0}^{\infty} \xi(s) d s=+\infty, \forall t>0
$$


And also, where $1<\sigma<3 / 2, \forall t_{0}>0$ fixed, $\exists C_{\sigma}(\sigma)>0$, such that

$$
\frac{t}{\left(1+\int_{t_{0}}^{t} \xi(s) d s\right)^{1 /(2(\sigma-1))}} \leq C_{\sigma}, \quad \forall t \geq t_{0} .
$$

(G3) we take

$$
\begin{aligned}
& f_{1}(u, v)=a|u+v|^{2(r+1)}(u+v)+b|u|^{r} u|v|^{r+2}, \\
& f_{2}(u, v)=a|u+v|^{2(r+1)}(u+v)+b|v|^{r} v|u|^{r+2},
\end{aligned}
$$

where $a, b>0$ and $r>-1$.

We have

$$
u f_{1}(u, v)+v f_{2}(u, v)=2(r+2) F(u, v), \forall(u, v) \in \mathbb{R}^{2},
$$

where

$$
\begin{aligned}
(\mathrm{G} 4) \mu_{2}, \mu_{4}:\left[\tau_{1}, \tau_{2}\right] & \longrightarrow \mathbb{R} \text { satisfying } \\
& \int_{\tau_{1}}^{\tau_{2}}\left|\mu_{2}(\mathrm{\varrho})\right| d \mathrm{\varrho}<\mu_{1}, \\
& \int_{\tau_{1}}^{\tau_{2}}\left|\mu_{4}(\mathrm{\varrho})\right| d \mathrm{\varrho}<\mu_{3} .
\end{aligned}
$$$$
F(u, v)=\frac{1}{2(r+2)}\left[a|u+v|^{2(r+2)}+2 b|u v|^{r+2}\right]
$$

Theorem 2. Assume (14) and $p<3$. Then, $\forall\left(u_{0}, v_{0}\right) \in V_{0}^{2}$, $\left(v_{1}, v_{2}\right) \in H^{2}$ and $\left(f_{0}, g_{0}\right) \in K^{2}$ problem (1) has a unique local solution

$$
(u, v, z, y) \in C\left(0, t_{*} ; V_{0}^{2} \times K^{2}\right) \cap C^{1}\left(0, t_{*} ; H^{2} \times K^{2}\right),
$$

for $t_{*}>0$ small enough.

Lemma 3. For $r>-1, \exists \eta>0$ such that $\forall u, v \in V \cap V_{0}(0, L)$, we have

$$
\|u+v\|_{L_{x}^{2(r+2)}}^{2(r+2)}+2\|u v\|_{L_{x}^{(r+2)}}^{(r+2)} \leq \eta\left(l_{1}\left\|u_{x}\right\|_{H}^{2}+l_{2}\left\|v_{x}\right\|_{H}^{2}\right)^{r+2}
$$

Proof. It is clear that by using the Minkowski inequality we get

$$
\|u+v\|_{L_{x}^{2(r+2)}}^{2} \leq 2\left(\|u\|_{L_{x}^{2(r+2)}}^{2}+\|v\|_{L_{x}^{2(r+2)}}^{2}\right)
$$

Also, Hölder's and Young's inequalities give us

$$
\begin{aligned}
\|u v\|_{L_{x}^{(r+2)}}^{(r+2)} & \leq\|u\|_{L_{x}^{2(r+2)}}\|v\|_{L_{x}^{2(r+2)}} \\
& \leq c\left(l_{1}\left\|u_{x}\right\|_{H}^{2}+l_{2}\left\|v_{x}\right\|_{H}^{2}\right) .
\end{aligned}
$$

By applying the embedding $V \cap V_{0}(0, L) \hookrightarrow L_{x}^{2(r+2)}(0, L)$ and (25), (27) gives (15).
Lemma 4. $\exists \Lambda_{1}, \Lambda_{2}>0$ such that

$\int_{0}^{L} x\left|f_{i}(u, v)\right|^{2} d x \leq \Lambda_{i}\left(l_{1} \int_{0}^{L} x u_{x}^{2} d x+l_{2} \int_{0}^{L} x v_{x}^{2} d x\right)^{2 r+3}, \forall x \in(0, L), i=1,2$.

Proof. We prove inequality for $f_{1}$ and the same result also holds for $f_{2}$.

It is clear that

$$
\begin{aligned}
\left|f_{1}(u, v)\right| & \leq C\left(|u+v|^{2 r+3}+|u|^{r+1}|v|^{r+2}\right) \\
& \leq C\left[|u|^{2 r+3}+|v|^{2 r+3}+|u|^{r+1}|v|^{r+2}\right] .
\end{aligned}
$$

By Young's inequality, with

$$
\begin{aligned}
q & =\frac{2 r+3}{r+1}, \\
q^{\prime} & =\frac{2 r+3}{r+2},
\end{aligned}
$$

we get

$$
|u|^{r+1}|v|^{r+2} \leq c_{1}|u|^{2 r+3}+c_{2}|v|^{2 r+3} .
$$

Therefore,

$$
\left|f_{1}(u, v)\right| \leq C\left[|u|^{2 r+3}+|v|^{2 r+3}\right] \text {. }
$$

Hence, by Poincaré's inequality and (11), we obtain

$$
\begin{aligned}
\int_{0}^{L} x\left|f_{i}(u, v)\right|^{2} d x & \leq C\left(\left\|u_{x}\right\|_{H}^{2(2 r+3)}+\left\|v_{x}\right\|_{H}^{2(2 r+3)}\right) \\
& \leq \Lambda_{1}\left(l_{1}\left\|u_{x}\right\|_{H}^{2}+l_{2}\left\|v_{x}\right\|_{H}^{2}\right)^{(2 r+3)}
\end{aligned}
$$

The proof of lemma is complete.

The energy function (see, e.g., $[8,19]$ and reference therein) is defined by

$$
\begin{aligned}
E(t)= & \frac{1}{2} \int_{0}^{L} x u_{t}^{2} d x+\frac{1}{2} \int_{0}^{L} x v_{t}^{2} d x+\frac{1}{2}\left(1-\int_{0}^{t} g_{1}(s) d s\right) \\
& \cdot \int_{0}^{L} x u_{x}^{2} d x+\frac{1}{2}\left(1-\int_{0}^{t} g_{2}(s) d s\right) \\
& \cdot \int_{0}^{L} x v_{x}^{2}(x, t) d x+\frac{1}{2} K(z, y) \\
& +\frac{1}{2}\left(g_{1} \circ u_{x}\right)(t)+\frac{1}{2}\left(g_{2} \circ v_{x}\right)(t)-\int_{0}^{L} F(u, v) d x,
\end{aligned}
$$


where

$$
\begin{aligned}
K(z, y)= & \int_{0}^{L} \int_{0}^{1} \int_{\tau_{1}}^{\tau_{2}} x \varrho\left(\left|\mu_{2}(\varrho)\right| z^{2}(x, \rho, \varrho, t)+\left|\mu_{4}(\varrho)\right| y^{2}(x, \rho, \varrho, t)\right) d \varrho d \rho d x, \\
& \left(g \circ u_{x}\right)(t)=\int_{0}^{L} \int_{0}^{t} x g(t-s)\left|u_{x}(x, t)-u_{x}(x, s)\right|^{2} d s d x .
\end{aligned}
$$

Lemma 5. Let $(u, v, z, y)$ be the solution of system (11); then, $E(t)$ is a nonincreasing function, that is, $\forall t \geq 0$

$$
\begin{aligned}
E^{\prime}(t) \leq & -d_{1} \int_{0}^{L} x u_{t}^{2} d x-d_{2} \int_{0}^{L} x v_{t}^{2} d x+\frac{1}{2}\left(g_{1}^{\prime} \circ u_{x}\right)(t)+\frac{1}{2}\left(g_{2}^{\prime} \circ v_{x}\right)(t) \\
& -\frac{1}{2} g_{1}(t) \int_{0}^{L} x u_{x}^{2} d x-\frac{1}{2} g_{2}(t) \int_{0}^{L} x v_{x}^{2} d x \leq 0,
\end{aligned}
$$

where

$$
\begin{gathered}
d_{1}=\mu_{1}-\left(\int_{\tau_{1}}^{\tau_{2}}\left|\mu_{2}(\mathrm{Q})\right| d \mathrm{Q}\right)>0, \\
d_{2}=\mu_{3}-\left(\int_{\tau_{1}}^{\tau_{2}}\left|\mu_{4}(\mathrm{Q})\right| d \mathrm{Q}\right)>0 .
\end{gathered}
$$

Proof. Multiplying equation $(11)_{1,2}$ by $x u_{t}, x v_{t}$, and integrating over $(0, L)$, we find

$$
\begin{aligned}
& \int_{0}^{L} x u_{t t} u_{t} d x-\int_{0}^{L}\left(x u_{x}\right)_{x} u_{t} d x+\mu_{1} \int_{0}^{L} x u_{t}^{2} d x \\
& \quad+\int_{0}^{L} x u_{t} \int_{\tau_{1}}^{\tau_{2}}\left|\mu_{2}(\varrho)\right| z(x, 1, \varrho, t) d \rho d x \\
& \quad+\int_{0}^{L} \int_{0}^{t} g_{1}(t-s)\left(x u_{x}(x, s)\right)_{x} d s u_{t} d x \\
& \quad+\int_{0}^{L} x v_{t t} v_{t} d x-\int_{0}^{L}\left(x v_{x}\right)_{x} v_{t} d x+\mu_{3} \int_{0}^{L} x v_{t}^{2} d x \\
& \quad+\int_{0}^{L} x v_{t} \int_{\tau_{1}}^{\tau_{2}}\left|\mu_{4}(\varrho)\right| y(x, 1, \varrho, t) d \varrho d x \\
& \quad+\int_{0}^{L} \int_{0}^{t} g_{2}(t-s)\left(x v_{x}(x, s)\right)_{x} d s v_{t} d x \\
& =\int_{0}^{L}\left[a|u+v|^{2(r+1)}(u+v)+b|u|^{r} u|v|^{r+2}\right] x u_{t} d x \\
& \quad+\int_{0}^{L}\left[a|u+v|^{2(r+1)}(u+v)+b|v|^{r} v|u|^{r+2}\right] x v_{t} d x .
\end{aligned}
$$

Using integration by parts, we get

$$
\begin{aligned}
& \int_{0}^{L} x u_{t t} u_{t} d x=\frac{1}{2} \frac{d}{d t}\left[\int_{0}^{L} x u_{t}^{2} d x\right], \\
& \int_{0}^{L} x v_{t t} v_{t} d x=\frac{1}{2} \frac{d}{d t}\left[\int_{0}^{L} x v_{t}^{2} d x\right],
\end{aligned}
$$

$$
\begin{aligned}
& -\int_{0}^{L}\left(x u_{x}\right)_{x} u_{t} d x=\frac{1}{2} \frac{d}{d t}\left[\int_{0}^{L} x u_{x}^{2} d x\right], \\
& -\int_{0}^{L}\left(x v_{x}\right)_{x} v_{t} d x=\frac{1}{2} \frac{d}{d t}\left[\int_{0}^{L} x v_{x}^{2} d x\right],
\end{aligned}
$$

$$
\begin{gathered}
\frac{1}{2(r+2)} \int_{0}^{L} x f_{1}(u, v) u u_{t} d x+\frac{1}{2(r+2)} \int_{0}^{L} x f_{2}(u, v) v v_{t} d x \\
=\frac{1}{2(r+2)} \frac{d}{d t} \int_{0}^{L}\left[a|u+v|^{2(r+2)}+2 b|u v|^{r+2}\right] x d x,
\end{gathered}
$$$$
\int_{0}^{L} \int_{0}^{t} g_{1}(t-s)\left(x u_{x}(s)\right)_{x} d s u_{t}(t) d x
$$$$
=\frac{1}{2} \frac{d}{d t}\left[\left(g_{1} \circ u_{x}\right)(t)-\int_{0}^{t} g_{1}(s) d s \int_{0}^{L} x u_{x}^{2} d x\right]
$$$$
-\frac{1}{2}\left(g_{1}^{\prime} \circ u_{x}\right)(t)+\frac{1}{2} g_{1}(t) \int_{0}^{L} x u_{x}^{2} d x,
$$

$$
\begin{aligned}
& \int_{0}^{L} \int_{0}^{t} g_{2}(t-s)\left(x v_{x}(s)\right)_{x} d s v_{t}(t) d x \\
& =\frac{1}{2} \frac{d}{d t}\left[\left(g_{2} \circ v_{x}\right)(t)-\int_{0}^{t} g_{2}(t) d s \int_{0}^{L} x v_{x}^{2} d x\right] \\
& \quad-\frac{1}{2}\left(g_{2}^{\prime} \circ v_{x}\right)(t)+\frac{1}{2} g_{2}(t) \int_{0}^{L} x v_{x}^{2} d x .
\end{aligned}
$$

Now, multiplying equation $(11)_{3}$ by $x z\left|\mu_{2}(\mathrm{e})\right|$ and integrating over $(0, L) \times(0,1) \times\left(\tau_{1}, \tau_{2}\right)$, we get

$$
\begin{aligned}
\frac{d}{d t} & \frac{1}{2} \int_{0}^{L} \int_{0}^{1} \int_{\tau_{1}}^{\tau_{2}} \mathrm{\varrho}\left|\mu_{2}(\mathrm{\varrho})\right| x z^{2} d \mathrm{\varrho} d \rho d x \\
= & -\int_{0}^{L} \int_{0}^{1} \int_{\tau_{1}}^{\tau_{2}}\left|\mu_{2}(\mathrm{\varrho})\right| x z z_{\rho} d \mathrm{\varrho} d \rho d x \\
= & -\frac{1}{2} \int_{0}^{L} \int_{0}^{1} \int_{\tau_{1}}^{\tau_{2}} x\left|\mu_{2}(\mathrm{\varrho})\right| \frac{d}{d \rho} z^{2} d \mathrm{\varrho} d \rho d x \\
= & \frac{1}{2} \int_{0}^{L} \int_{\tau_{1}}^{\tau_{2}} x\left|\mu_{2}(\mathrm{\varrho})\right|\left((z(x, 0, \mathrm{\varrho}, t))^{2}\right. \\
& \left.-(z(x, 1, \mathrm{\varrho}, t))^{2}\right) d \mathrm{\varrho} d x \\
= & \frac{1}{2} \int_{\tau_{1}}^{\tau_{2}}\left|\mu_{2}(\mathrm{\varrho})\right| d \mathrm{\varrho} \int_{0}^{L}\left|x u_{t}\right|^{2} d x \\
& -\frac{1}{2} \int_{0}^{L} \int_{\tau_{1}}^{\tau_{2}} x\left|\mu_{2}(\mathrm{\varrho})\right|(z(x, 1, \mathrm{\varrho}, t))^{2} d \mathrm{\varrho} d x .
\end{aligned}
$$

Similarly, by multiplying equation (11) ${ }_{4}$ by $x y\left|\mu_{4}(\mathrm{Q})\right|$ 
and integrating over $(0, L) \times(0,1) \times\left(\tau_{1}, \tau_{2}\right)$, we get

$$
\begin{aligned}
& \frac{d}{d t} \frac{1}{2} \int_{0}^{L} \int_{0}^{1} \int_{\tau_{1}}^{\tau_{2}} \mathrm{\varrho}\left|\mu_{4}(\mathrm{\varrho})\right| x y^{2} d \mathrm{\varrho} d \rho d x \\
& =\frac{1}{2}\left(\int_{\tau_{1}}^{\tau_{2}}\left|\mu_{4}(\mathrm{\varrho})\right| d \mathrm{\varrho}\right) \int_{0}^{L} x v_{t}^{2} d x \\
& \quad-\frac{1}{2} \int_{0}^{L} \int_{\tau_{1}}^{\tau_{2}} x\left|\mu_{4}(\mathrm{\varrho})\right| y^{2}(x, 1, \mathrm{\varrho}, t) d \mathrm{\varrho} d x .
\end{aligned}
$$

Using Young's and Cauchy-Schwartz inequalities, we have

$$
\begin{aligned}
-\int_{0}^{L} x u_{t} \int_{\tau_{1}}^{\tau_{2}}\left|\mu_{2}(\varrho)\right| z(x, 1, \varrho, t) d \varrho d x \\
\leq \frac{1}{2}\left(\int_{\tau_{1}}^{\tau_{2}}\left|\mu_{2}(\mathrm{\varrho})\right| d \varrho\right) \int_{0}^{L} x u_{t}^{2} d x \\
\quad+\frac{1}{2} \int_{0}^{L} \int_{\tau_{1}}^{\tau_{2}}\left|\mu_{2}(\varrho)\right| x z^{2}(x, 1, \varrho, t) d \varrho d x .
\end{aligned}
$$

Similarly, we get

$$
\begin{aligned}
& -\int_{0}^{L} x v_{t} \int_{\tau_{1}}^{\tau_{2}}\left|\mu_{4}(\mathrm{\varrho})\right| y(x, 1, \varrho, t) d \varrho d x \\
& \leq \frac{1}{2}\left(\int_{\tau_{1}}^{\tau_{2}}\left|\mu_{4}(\mathrm{\varrho})\right| d \varrho\right) \int_{0}^{L} x v_{t}^{2} d x \\
& \quad+\frac{1}{2} \int_{0}^{L} \int_{\tau_{1}}^{\tau_{2}}\left|\mu_{4}(\mathrm{\varrho})\right| x y^{2}(x, 1, \varrho, t) d \varrho d x .
\end{aligned}
$$

By combining (39), (40), (41), (42), (43), (45), (46), (47), (48), (49), and (50) in (38), we get (34) and (36).

\section{Global Existence}

In this section, we showed the global existence of the solutions of the system (11).

First, introducing the following notation

$$
\begin{aligned}
I(t):= & I(u(t), v(t))=\left(1-\int_{0}^{t} g_{1}(s) d s\right) \int_{0}^{L} x u_{x}^{2} d x+\left(g_{1} \circ u_{x}\right)(t) \\
& +\left(1-\int_{0}^{t} g_{2}(s) d s\right) \int_{0}^{L} x v_{x}^{2} d x+\left(g_{2} \circ v_{x}\right)(t) \\
& +K(z, y)-2(r+2) \int_{0}^{L} x\left[a|u+v|^{2(r+2)}+2 b|u v|^{r+2}\right] d x
\end{aligned}
$$

$$
\begin{aligned}
J(t):= & J(u(t), v(t))=\frac{1}{2}\left(1-\int_{0}^{t} g_{1}(s) d s\right) \int_{0}^{L} x u_{x}^{2} d x \\
& +\frac{1}{2}\left(g_{1} \circ u_{x}\right)(t)+\frac{1}{2}\left(1-\int_{0}^{t} g_{2}(s) d s\right) \int_{0}^{L} x v_{x}^{2} d x \\
& +\frac{1}{2}\left(g_{2} \circ v_{x}\right)(t)+\frac{1}{2} K(z, y)-\int_{0}^{L} x\left[a|u+v|^{2(r+2)}+2 b|u v|^{r+2}\right] d x,
\end{aligned}
$$

note that

$$
E(t)=J(t)+\frac{1}{2} \int_{0}^{L} x u_{t}^{2} d x+\frac{1}{2} \int_{0}^{L} x v_{t}^{2} d x
$$

Lemma 6. Assume that (24), (14), (15), (16), (17), and (22) hold, and $\forall\left(u_{0}, v_{0}\right) \in V_{0}^{2},\left(u_{1}, v_{1}\right) \in H^{2}$ and $\left(f_{0}, g_{0}\right)$ $\in L_{x}^{2}\left((0, L),(0,1),\left(\tau_{1}, \tau_{2}\right)\right)$ satisfying

$$
I(0)>0, \beta:=\eta\left(\frac{2(r+2)}{(r+1)} E(0)\right)^{r+1}<1 .
$$

Then, $\exists t_{*}>0$ such that

$$
I(t)>0, \forall t \in\left[0, t_{*}\right)
$$

where

$$
E(0)=J(0)+\frac{1}{2} \int_{0}^{L} x u_{1}^{2} d x+\frac{1}{2} \int_{0}^{L} x v_{1}^{2} d x
$$

Proof. As $I(0)>0$, then by continuity of $I(t), \exists T_{m} \leq t_{*}$ such that $I(t) \geq 0, \forall t \in\left[0, T_{m}\right)$; this implies that we have a maximum time value noting $T_{m}$ such that

$$
\left\{I\left(T_{m}\right)=0 \text { and } I(t)>0, \quad \text { for all } 0 \leq t<T_{m}\right\} \text {. }
$$

This, with (51), (52), and (14), we have

$$
\begin{aligned}
J(t)= & \frac{r+1}{2(r+2)}\left[\left(1-\int_{0}^{t} g_{1}(s) d s\right) \int_{0}^{L} x u_{x}^{2} d x\right. \\
& \left.+\left(1-\int_{0}^{t} g_{2}(s) d s\right) \int_{0}^{L} x v_{x}^{2} d x\right] \\
& +\frac{r+1}{2(r+2)}\left[\left(g_{1} \circ u_{x}\right)(\mathrm{t})+\left(g_{2} \circ v_{x}\right)(t)+K(z, y)\right] \\
& +\frac{1}{2(r+2)} I(t) \\
\geq & \frac{r+1}{2(r+2)}\left[\left(l_{1} \int_{0}^{L} x u_{x}^{2} d x+l_{2} \int_{0}^{L} x v_{x}^{2} d x\right)\right. \\
& \left.+\left(g_{1} \circ u_{x}\right)(t)+\left(g_{2} \circ v_{x}\right)(t)+K(z, y)\right]
\end{aligned}
$$

Hence,

$$
\begin{aligned}
& l_{1} \int_{0}^{L} x u_{x}^{2} d x+l_{2} \int_{0}^{L} x v_{x}^{2} d x \\
& \quad \leq \frac{2(r+2)}{r+1} J(t) \\
& \quad \leq \frac{2(r+2)}{r+1} E(t) \\
& \quad \leq \frac{2(r+2)}{r+1} E(0), \quad \forall t \in\left[0, T_{m}\right) .
\end{aligned}
$$


By (24) and (54), we get

$$
\begin{aligned}
& 2(r+2) \int_{0}^{L} F\left(u\left(T_{m}\right), v\left(T_{m}\right)\right) d x \leq \eta\left(l_{1} \int_{0}^{L} x u_{x}^{2} d x+l_{2} \int_{0}^{L} x v_{x}^{2} d x\right)^{r+2} \\
& \leq \eta\left(\frac{2(r+2)}{r+1} E(0)\right)^{r+1}\left(l_{1} \int_{0}^{L} x u_{x}^{2} d x+l_{2} \int_{0}^{L} x v_{x}^{2} d x\right) \\
& =\beta\left(l_{1} \int_{0}^{L} x u_{x}^{2} d x+l_{2} \int_{0}^{L} x v_{x}^{2} d x\right) \\
& <\left(1-\int_{0}^{t} g_{1}(s) d s\right) \int_{0}^{L} x u_{x}^{2} d x \\
& \quad+\left(1-\int_{0}^{t} g_{2}(s) d s\right) \int_{0}^{L} x v_{x}^{2} d x \\
& \quad+\left(g_{1} \circ u_{x}\right)(t)+\left(g_{2} \circ v_{x}\right)(t)+K(z, y) .
\end{aligned}
$$

Hence,

$$
\begin{aligned}
& \left(1-\int_{0}^{t} g_{1}(s) d s\right) \int_{0}^{L} x u_{x}^{2} d x+\left(1-\int_{0}^{t} g_{2}(s) d s\right) \int_{0}^{L} x v_{x}^{2} d x \\
& \quad+\left(g_{1} \circ u_{x}\right)(t)+\left(g_{2} \circ v_{x}\right)(t)+K(z, y) \\
& \quad-2(r+2) \int_{0}^{L} x F(u, v) d x>0 .
\end{aligned}
$$

This proves that $I(t)>0, \forall t \in\left[0, T_{m}\right)$. By repeating the procedure, $T_{m}$ is extended to $t_{*}$.

Theorem 7. Let (14), (15), (16), (17), (22), and (24) hold. Then, $\forall\left(u_{0}, v_{0}\right) \in V_{0}^{2},\left(u_{1}, v_{1}\right) \in H^{2}$, and $\left(f_{0}, g_{0}\right) \in L_{x}^{2}((0, L)$, $\left.(0,1),\left(\tau_{1}, \tau_{2}\right)\right)$ satisfying (54) the solution of system (11) is bounded and global.

Proof. To prove that $\left\|u_{x}\right\|_{H}^{2}+\left\|v_{x}\right\|_{H}^{2}+\left\|u_{t}\right\|_{H}^{2}+\left\|v_{t}\right\|_{H}^{2}+$ $\|z\|_{K, \mu_{2}}^{2}+\|y\|_{K, \mu_{4}}^{2}$ is bounded independently of $t$, using (36) yields

$$
E(0) \geq E(t)
$$

Using (52), we find

$$
\begin{aligned}
-2(r+2) \int_{0}^{L} x\left[a|u+v|^{2(r+2)}+2 b|u v|^{r+2}\right] d x \\
=I(t)-\left(1-\int_{0}^{t} g_{1}(s) d s\right) \int_{0}^{L} x u_{x}^{2} d x \\
-\left(1-\int_{0}^{t} g_{2}(s) d s\right) \int_{0}^{L} x v_{x}^{2} d x \\
-\left(g_{1} \circ u_{x}\right)(t)-\left(g_{2} \circ v_{x}\right)(t)-K(z, y) .
\end{aligned}
$$

By using (62) in (63), we get

$$
\begin{aligned}
E(0) \geq E(t)=\frac{1}{2} \int_{0}^{L} x u_{t}^{2} d x+\frac{1}{2} \int_{0}^{L} x v_{t}^{2} d x \\
+\frac{1}{2}\left(1-\int_{0}^{t} g_{1}(s) d s\right) \int_{0}^{L} x u_{x}^{2} d x \\
+\frac{1}{2}\left(1-\int_{0}^{t} g_{2}(s) d s\right) \int_{0}^{L} x v_{x}^{2}(x, t) d x \\
+\frac{1}{2}\left(g_{1} \circ u_{x}\right)(t)+\frac{1}{2}\left(g_{2} \circ v_{x}\right)(t) \\
+\frac{1}{2} K(z, y)+I(t),
\end{aligned}
$$

and using (14), (15), and (54) in (64), we get

$$
\begin{aligned}
E(0) \geq & E(t) \geq \frac{1}{2} \int_{0}^{L} x u_{t}^{2} d x+\frac{1}{2} \int_{0}^{L} x v_{t}^{2} d x \\
& +\left(\frac{r+1}{2(r+2)}\right)\left\{l_{1} \int_{0}^{L} x u_{x}^{2} d x+l_{2} \int_{0}^{L} x v_{x}^{2} d x+K(z, y)\right\} \\
\geq & \mu_{0}\left(\int_{0}^{L} x u_{t}^{2} d x+\int_{0}^{L} x v_{t}^{2} d x+\int_{0}^{L} x u_{x}^{2} d x\right. \\
& \left.+\int_{0}^{L} x v_{x}^{2} d x+K(z, y)\right) .
\end{aligned}
$$

So

$\left\|u_{x}\right\|_{H}^{2}+\left\|v_{x}\right\|_{H}^{2}+\left\|u_{t}\right\|_{H}^{2}+\left\|v_{t}\right\|_{H}^{2}+\|z\|_{K, \mu_{2}}^{2}+\|y\|_{K, \mu_{4}}^{2} \leq \mu E(0) / \mu:=\frac{1}{\mu_{0}}$,

where

$$
\mu_{0}:=\min \left\{\frac{1}{2}, \frac{(r+1)}{2(r+2)} l_{1}, \frac{(r+1)}{2(r+2)} l_{2}, \frac{(r+1)}{2(r+2)}\right\}
$$

Hence, the solution of system (11) is bounded and global.

\section{Decay of Solutions}

In this section, the decay result is showed by using several lemmas.

As, we let

$$
F(t):=E(t)+\varepsilon_{1} \Phi(t)+\varepsilon_{2} \chi(t)+\varepsilon_{3} \Psi(t),
$$

where $\varepsilon_{1}, \varepsilon_{2}, \varepsilon_{3}>0$, and

$$
\Phi(t):=\xi(t) \int_{0}^{L} x u_{t} u d x+\xi(t) \int_{0}^{L} x v_{t} v d x
$$




$$
\begin{aligned}
\chi(t):= & -\xi(t) \int_{0}^{L} x u_{t} \int_{0}^{t} g_{1}(t-s)(u(t)-u(s)) d s d x \\
& -\xi(t) \int_{0}^{L} x v_{t} \int_{0}^{t} g_{2}(t-s)(v(t)-v(s)) d s d x \\
\Psi(z, y):= & \xi(t) \int_{0}^{L} \int_{0}^{1} \int_{\tau_{1}}^{\tau_{2}} x \varrho e^{-\rho \varrho}\left(\left|\mu_{2}(\varrho)\right| z^{2}+\left|\mu_{4}(\varrho)\right| y^{2}\right) d \varrho d \rho d x .
\end{aligned}
$$

Lemma 8. There exist $\alpha_{1}, \alpha_{2}>0$, such that

$$
\alpha_{1} F(t) \leq E(t) \leq \alpha_{2} F(t)
$$

for $\varepsilon_{1}, \varepsilon_{2}$, and $\varepsilon_{3}$ small enough.

Proof. Using the inequality of Young and the Poincaré-type inequality and $0<\xi(t) \leq \xi(0)$, we find

$$
\varepsilon_{1} \xi(t) \int_{0}^{L} x u_{t} u d x \leq \frac{\varepsilon_{1}}{2} \xi(0) \int_{0}^{L} x u_{t}^{2} d x+\frac{\varepsilon_{1}}{2} C_{p} \xi(0) \int_{0}^{L} x u_{x}^{2} d x
$$

$$
\varepsilon_{1} \xi(t) \int_{0}^{L} x v_{t} v d x \leq \frac{\varepsilon_{1}}{2} \xi(0) \int_{0}^{L} x v_{t}^{2} d x+\frac{\varepsilon_{1}}{2} C_{p} \xi(0) \int_{0}^{L} x v_{x}^{2} d x,
$$

$$
\begin{aligned}
& -\varepsilon_{2} \xi(t) \int_{0}^{L} x u_{t} \int_{0}^{t} g_{1}(t-s)(u(t)-u(s)) d s d x \\
& \quad \leq \frac{\varepsilon_{2}}{2} \xi(0) \int_{0}^{L} x u_{t}^{2} d x+\frac{\varepsilon_{2}}{2} C_{p} \xi(0)\left(1-l_{1}\right)\left(g_{1} \circ u_{x}\right)(t),
\end{aligned}
$$

$$
\begin{aligned}
& -\varepsilon_{2} \xi(t) \int_{0}^{L} x v_{t} \int_{0}^{t} g_{2}(t-s)(v(t)-v(s)) d s d x \\
& \quad \leq \frac{\varepsilon_{2}}{2} \xi(0) \int_{0}^{L} x v_{t}^{2} d x+\frac{\varepsilon_{2}}{2} C_{p} \xi(0)\left(1-l_{2}\right)\left(g_{2} \circ v_{x}\right)(t),
\end{aligned}
$$

$\Psi(z, y) \leq \xi(0) \int_{0}^{L} \int_{0}^{1} \int_{\tau_{1}}^{\tau_{2}} x \varrho\left(\left|\mu_{2}(\mathrm{\varrho})\right| z^{2}+\left|\mu_{4}(\mathrm{\varrho})\right| y^{2}\right) d \varrho d \rho d x$,
A combination of (73), (74), (75), (76), and (77) in (68) gives

$$
\begin{aligned}
F(t) \leq & E(t)+\left(\frac{\varepsilon_{1+} \varepsilon_{2}}{2}\right) \xi(0) \int_{0}^{L} x u_{t}^{2} d x+\left(\frac{\varepsilon_{1}+\varepsilon_{2}}{2}\right) \xi(0) \int_{0}^{L} x v_{t}^{2} d x \\
& +\frac{\varepsilon_{1}}{2} C_{p} \xi(0) \int_{0}^{L} x u_{x}^{2} d x+\frac{\varepsilon_{1}}{2} C_{p} \xi(0) \int_{0}^{L} x v_{x}^{2} d x \\
& +\frac{\varepsilon_{2}}{2} C_{p} \xi(0)\left(1-l_{1}\right)\left(g_{1} \circ u_{x}\right)(t) \\
& +\frac{\varepsilon_{2}}{2} C_{p} \xi(0)\left(1-l_{2}\right)\left(g_{2} \circ v_{x}\right)(t) \\
& +\varepsilon_{3} \xi(0) \int_{0}^{L} \int_{0}^{1} \int_{\tau_{1}}^{\tau_{2}} x \rho\left(\left|\mu_{2}(\mathrm{\varrho})\right| z^{2}+\left|\mu_{4}(\mathrm{\varrho})\right| y^{2}\right) d \mathrm{\varrho} d \rho d x .
\end{aligned}
$$

Then, $\exists \alpha_{1}>0$, for $\varepsilon_{1}, \varepsilon_{2}$, and $\varepsilon_{3}$ small enough, such that

$$
F(t) \leq \frac{1}{\alpha_{1}} E(t)
$$

Similarly, thanks to the inequalities of Young and Poincaré-type and using $0<\xi(t) \leq \xi(0)$ gives

$$
\varepsilon_{1} \xi(t) \int_{0}^{L} x u_{t} u d x \geq \frac{-\varepsilon_{1}}{2} \xi(0) \int_{0}^{L} x u_{t}^{2} d x-\frac{\varepsilon_{1}}{2} C_{p} \xi(0) \int_{0}^{L} x u_{x}^{2} d x,
$$

$$
\begin{gathered}
\varepsilon_{1} \xi(t) \int_{0}^{L} x v_{t} v d x \geq \frac{-\varepsilon_{1}}{2} \xi(0) \int_{0}^{L} x v_{t}^{2} d x-\frac{\varepsilon_{1}}{2} C_{p} \xi(0) \int_{0}^{L} x v_{x}^{2} d x \\
-\varepsilon_{2} \xi(t) \int_{0}^{L} x u_{t} \int_{0}^{t} g_{1}(t-s)(u(t)-u(s)) d s d x \\
\geq \frac{-\varepsilon_{2}}{2} \xi(0) \int_{0}^{L} x u_{t}^{2} d x-\frac{\varepsilon_{2}}{2} C_{p} \xi(0)\left(1-l_{1}\right)\left(g_{1} \circ u_{x}\right)(t), \\
-\varepsilon_{2} \xi(t) \int_{0}^{L} x v_{t} \int_{0}^{t} g_{2}(t-s)(v(t)-v(s)) d s d x \\
\geq \frac{-\varepsilon_{2}}{2} \xi(0) \int_{0}^{L} x v_{t}^{2} d x-\frac{\varepsilon_{2}}{2} C_{p} \xi(0)\left(1-l_{2}\right)\left(g_{2} \circ v_{x}\right)(t),
\end{gathered}
$$

and

$-\varepsilon_{3} \Psi(z, y) \geq-\varepsilon_{3} \xi(0) \int_{0}^{L} \int_{0}^{1} \int_{\tau_{1}}^{\tau_{2}} x \mathrm{\varrho}\left(\left|\mu_{2}(\mathrm{\varrho})\right| z^{2}+\left|\mu_{4}(\rho \mathrm{\varrho})\right| y^{2}\right) d \varrho d \rho d x$.

where $C_{p}>0$. 
By combining (80), (81), (82), (83), and (84) in (68), we find

$$
\begin{aligned}
F(t) \geq & E(t)-\left(\frac{\varepsilon_{1+} \varepsilon_{2}}{2}\right) \xi(0) \int_{0}^{L} x u_{t}^{2} d x-\left(\frac{\varepsilon_{1}+\varepsilon_{2}}{2}\right) \xi(0) \int_{0}^{L} x v_{t}^{2} d x \\
& -\frac{\varepsilon_{1}}{2} C_{p} \xi(0) \int_{0}^{L} x u_{x}^{2} d x-\frac{\varepsilon_{1}}{2} C_{p} \xi(0) \int_{0}^{L} x v_{x}^{2} d x \\
& -\frac{\varepsilon_{2}}{2} C_{p} \xi(0)\left(1-l_{1}\right)\left(g_{1} \circ u_{x}\right)(t) \\
& -\frac{\varepsilon_{2}}{2} C_{p} \xi(0)\left(1-l_{2}\right)\left(g_{2} \circ v_{x}\right)(t) \\
& -\varepsilon_{3} \xi(0) \int_{0}^{L} \int_{0}^{1} \int_{\tau_{1}}^{\tau_{2}} x \rho\left(\left|\mu_{2}(\varrho)\right| z^{2}+\left|\mu_{4}(\mathrm{\varrho})\right| y^{2}\right) d \varrho d \rho d x .
\end{aligned}
$$

Then, $\exists \alpha_{2}>0$, for $\varepsilon_{1}, \varepsilon_{2}$, and $\varepsilon_{3}$ small enough, such that

$$
F(t) \geq \frac{1}{\alpha_{2}} E(t)
$$

This completes the proof.

Lemma 9. For $\sigma>1$ and $0<\theta<1$, we have

$$
\begin{aligned}
& \int_{0}^{t} g(t-s)\|w(s)\|^{2} d s \\
& \leq\left(\int_{0}^{t} g^{1-\theta}(t-s)\|w(s)\|^{2} d s\right)^{1 / \sigma} \\
& \quad \times\left(\int_{0}^{t} g^{(\sigma-1+\theta) / \sigma-1}(t-s)\|w(s)\|^{2} d s\right)^{(\sigma-1) / \sigma},
\end{aligned}
$$

$\forall w \in H$.

Proof. It suffices to note that

$$
\begin{aligned}
\int_{0}^{t} g(t-s)\|w(s)\|^{2} d s= & \int_{0}^{t} g^{(1-\theta) / r}(t-s)\|w(s)\|^{2 / r} g^{(\sigma-1+\theta) / \sigma} \\
& \cdot(t-s)\|w(s)\|^{(2(\sigma-1)) / \sigma} d s
\end{aligned}
$$

using Hỏlder's inequality for

$$
\begin{aligned}
& p=\sigma, \\
& q=\frac{\sigma}{\sigma-1}, \quad r>1 .
\end{aligned}
$$

This completes the proof.

Lemma 10. Let $v \in L^{\infty}((0, T) ; H)$ be such that $v_{x} \in L^{\infty}((0$, $t) ; H)$ and $g$ be a continuous function on $[0, T]$ and suppose that $0<\theta<1$ and $\rho>1$. Then, $\exists C>0$ so that

$$
\begin{aligned}
& \int_{0}^{t} g(t-s)\left\|v_{x}(., t)-v_{x}(., s)\right\|_{H}^{2} d s \\
& \leq C\left(\sup _{0<s<T}\|v(., s)\|_{H}^{2} \int_{0}^{t} g^{1-\theta}(s) d s\right)^{(\rho-1) /(\rho-1+\theta)} \\
& \quad \times\left(\int_{0}^{t} g^{\rho}(t-s)\left\|v_{x}(., t)-v_{x}(., s)\right\|_{H}^{2} d s\right)^{\theta /(\rho-1+\theta)} .
\end{aligned}
$$

Proof. By applying Lemma 8 with $\sigma=(\rho-1+\theta) /(\rho-1)$ gives

$$
\begin{aligned}
& \int_{0}^{t} g(t-s)\left\|v_{x}(., t)-v_{x}(., s)\right\|_{H}^{2} d s \\
& \leq\left(\int_{0}^{t} g^{1-\theta}(t-s)\left\|v_{x}(., t)-v_{x}(., s)\right\|_{H}^{2} d s\right)^{(\rho-1) /(\rho-1+\theta)} \\
& \quad \times\left(\int_{0}^{t} g^{\rho}(t-s)\left\|v_{x}(., t)-v_{x}(., s)\right\|_{H}^{2}\right)^{\theta /(\rho-1+\theta)} .
\end{aligned}
$$

We also have

$$
\int_{0}^{t} g^{1-\theta}(t-s)\left\|v_{x}(., t)-v_{x}(., s)\right\|_{H}^{2} d s \leq C \sup _{0<s<T}\left\|v_{x}(., s)\right\|_{H}^{2} \int_{0}^{t} g^{1-\theta}(s) d s,
$$

by combining (82) and (83). This completes the proof.

Lemma 11. Suppose that $v \in L^{\infty}((0, T) ; H)$ be such that $v_{x}$ $\in L^{\infty}((0, T) ; H)$ and $g$ be a continuous function on $[0, T]$ and assume $\rho>1$. Then, $\exists C>0$ so that

$$
\begin{aligned}
& \int_{0}^{t} g(t-s)\left\|v_{x}(., t)-v_{x}(., s)\right\|_{H}^{2} d s \\
& \leq c\left(t\left\|v_{x}(., t)\right\|_{H}^{2}+\int_{0}^{t}\left\|v_{x}(., s)\right\|_{H}^{2} d s\right)^{(\rho-1) / \rho} \\
& \quad \times\left(\int_{0}^{t} g^{\rho}(t-s)\left\|v_{x}(., t)-v_{x}(., s)\right\|_{H}^{2} d s\right)^{1 / \rho} .
\end{aligned}
$$

Proof. By using (82) for $\theta=1$ gives

$$
\begin{aligned}
& \int_{0}^{t} g(t-s)\left\|v_{x}(., t)-v_{x}(., s)\right\|_{H}^{2} d s \\
& \leq\left(\int_{0}^{t}\left\|v_{x}(., t)-v_{x}(., s)\right\|_{H}^{2} d s\right)^{(\rho-1) / \rho} \\
& \quad \times\left(\int_{0}^{t} g^{\rho}(t-s)\left\|v_{x}(., t)-v_{x}(., s)\right\|_{H}^{2} d s\right)^{1 / \rho},
\end{aligned}
$$


where

$$
\int_{0}^{t}\left\|v_{x}(., t)-v_{x}(., s)\right\|_{H}^{2} d s \leq 2 t\left\|v_{x}(., t)\right\|_{H}^{2}+2 \int_{0}^{t}\left\|v_{x}(., s)\right\|_{H}^{2} d s,
$$

to obtain (93). Hence, this ends the proof.

Lemma 12. Suppose that $r$ satisfies (15) and (52) hold. Then, the functional $\Phi(t)$, given by (69), satisfies

$$
\begin{aligned}
\Phi^{\prime}(t) \leq & \left(1+\frac{l}{2 \delta}+\frac{\mu_{1}}{2 \delta_{1}}\right) \xi(t) \int_{0}^{L} x u_{t}^{2} d x \\
& +\left(1+\frac{l}{2 \delta}+\frac{\mu_{3}}{2 \delta_{2}}\right) \xi(t) \int_{0}^{L} x v_{t}^{2} d x-\xi(t) \\
& \cdot\left(\frac{l_{1}-C_{p}\left(\delta l-2 \delta_{1} \mu_{1}\right)}{2}\right) \int_{0}^{L} x u_{x}^{2} d x-\xi(t) \\
& \cdot\left(\frac{l_{2}-C_{p}\left(\delta l-2 \delta_{2} \mu_{3}\right)}{2}\right) \int_{0}^{L} x v_{x}^{2} d x \\
& +\frac{\xi(t)}{2 l_{1}}\left(\int_{0}^{t} g_{1}^{2-\sigma}(s) d s\right)\left(g_{1}^{\sigma} \circ u_{x}\right)(t)+\frac{\xi(t)}{2 l_{2}} \\
& \cdot\left(\int_{0}^{t} g_{2}^{2-\sigma}(s) d s\right)\left(g_{2}^{\sigma} \circ v_{x}\right)(t) \\
& +\frac{\xi(t)}{2 \delta_{1}} \int_{0}^{L} \int_{\tau_{1}}^{\tau_{2}} x\left|\mu_{2}(\varrho)\right| z^{2}(x, 1, \varrho, t) d \varrho d x \\
& +\frac{\xi(t)}{2 \delta_{2}} \int_{0}^{L} \int_{\tau_{1}}^{\tau_{2}} x\left|\mu_{4}(\mathrm{\varrho})\right| y^{2}(x, 1, \varrho, t) d \mathrm{\varrho} d x \\
& +\frac{\xi(t)}{2(r+2)}\left[a|u+v|^{2(r+2)}+2 b|u v|^{r+2}\right] d x .
\end{aligned}
$$

For any $\delta, \delta_{1}, \delta_{2}>0$.

Proof. The derivation of (11) gives

$$
\begin{aligned}
\Phi^{\prime}(t)= & \xi^{\prime}(t) \int_{0}^{L} x u_{t} u d x+\xi(t) \int_{0}^{L} x u_{t}^{2} d x+\xi(t) \int_{0}^{L} x u_{t t} u d x \\
& +\xi^{\prime}(t) \int_{0}^{L} x v_{t} v d x+\xi(t) \int_{0}^{L} x v_{t}^{2} d x+\xi(t) \int_{0}^{L} x v_{t t} v d x \\
= & \xi^{\prime}(t) \int_{0}^{L} x u_{t} u d x+\xi(t) \int_{0}^{L} x u_{t}^{2} d x-\xi(t) \int_{0}^{L} x u_{x}^{2} d x \\
& -\xi(t) \mu_{1} \int_{0}^{L} x u u_{t} d x-\xi(t) \int_{0}^{L} x u \int_{\tau_{1}}^{\tau_{2}}\left|\mu_{2}(\mathrm{\varrho})\right| z^{2}(x, 1, \mathrm{\varrho}, t) d \mathrm{\varrho} d x \\
& +\xi(t) \int_{0}^{L} x u_{x} \int_{0}^{t} g_{1}(t-s) u_{x}(s) d s d x+\xi^{\prime}(t) \int_{0}^{L} x v_{t} v d x \\
& +\xi(t) \int_{0}^{L} x v_{t}^{2} d x-\xi(t) \int_{0}^{L} x v_{x}^{2} d x-\xi(t) \mu_{3} \int_{0}^{L} x v v_{t} d x \\
& -\xi(t) \int_{0}^{L} x v \int_{\tau_{1}}^{\tau_{2}}\left|\mu_{4}(\mathrm{\varrho})\right| y^{2}(x, 1, \varrho, t) d \mathrm{\varrho} d x \\
& +\xi(t) \int_{0}^{L} x v_{x} \int_{0}^{t} g_{2}(t-s) v_{x}(s) d s d x \\
& +\frac{\xi(t)}{2(r+2)}\left[a|u+v|^{2(r+2)}+2 b|u v|^{r+2}\right] d x .
\end{aligned}
$$

By Young's and Poincaré inequalities and (14) and (15), we find

$$
\begin{aligned}
& \xi(t) \int_{0}^{L} x u_{x}(t)\left(\int_{0}^{t} g_{1}(t-s) u_{x}(s) d s\right) d x \\
& \leq \frac{\xi(t)}{2} \int_{0}^{L} x u_{x}^{2} d x+\frac{\xi(t)}{2} \int_{0}^{L} x\left(\int _ { 0 } ^ { t } g _ { 1 } ( t - s ) \left(\mid u_{x}(s)\right.\right. \\
& \left.\left.\quad-u_{x}(t)|+| u_{x}(t) \mid\right) d s\right)^{2} d x \\
& \leq \frac{\xi(t)}{2} \int_{0}^{L} x u_{x}^{2} d x+\frac{\xi(t)}{2}\left(1+\eta_{1}\right)\left(1-l_{1}\right)^{2} \int_{0}^{L} x u_{x}^{2}(t) d x \\
& \quad+\frac{\xi(t)}{2}\left(1+\frac{1}{\eta_{1}}\right)\left(\int_{0}^{t} g_{1}^{2-\sigma}(s) d s\right)\left(g_{1}^{\sigma} \circ u_{x}\right)(t) \\
& =\xi(t)\left(\frac{1+\left(1+\eta_{1}\right)\left(1-l_{1}\right)^{2}}{2}\right) \int_{0}^{L} x u_{x}^{2} d x \\
& \quad+\frac{\xi(t)}{2}\left(1+\frac{1}{\eta_{1}}\right)\left(\int_{0}^{t} g_{1}^{2-\sigma}(s) d s\right)\left(g_{1}^{\sigma} \circ u_{x}\right)(t) \\
& \quad+\frac{\xi(t)}{r+2} \int_{0}^{L}\left[a|u+v|^{2(r+2)}+2 b|u v|^{r+2}\right] d x .
\end{aligned}
$$

Similarly, we get

$$
\begin{aligned}
& \int_{0}^{L} x v_{x}(t)\left(\int_{0}^{t} g_{1}(t-s) v_{x}(s) d s\right) d x \\
& \leq \xi(t)\left(\frac{1+\left(1+\eta_{2}\right)\left(1-l_{2}\right)^{2}}{2}\right) \int_{0}^{L} x v_{x}^{2} d x \\
& \quad+\frac{\xi(t)}{2}\left(1+\frac{1}{\eta_{2}}\right)\left(\int_{0}^{t} g_{2}^{2-\sigma}(s) d s\right)\left(g_{2}^{\sigma} \circ v_{x}\right)(t)
\end{aligned}
$$

$$
\forall \eta_{1}, \eta_{2}>0 \text {. As we have }
$$

$$
\begin{aligned}
\xi^{\prime}(t) \int_{0}^{L} x u_{t} u d x & \leq \frac{\xi(t)}{2}\left|\frac{\xi^{\prime}(t)}{\xi(t)}\right|\left(C_{p} \delta \int_{0}^{L} x u_{x}^{2} d x+\frac{1}{\delta} \int_{0}^{L} x u_{t}^{2} d x\right) \\
& \leq \frac{\xi(t)}{2}\left(C_{p} l \delta \int_{0}^{L} x u_{x}^{2} d x+\frac{l}{\delta} \int_{0}^{L} x u_{t}^{2} d x\right), \forall \delta>0,
\end{aligned}
$$

and similarly, we find

$$
\xi^{\prime}(t) \int_{0}^{L} x v_{t} v d x \leq \frac{\xi(t)}{2}\left(C_{p} l \delta \int_{0}^{L} x v_{x}^{2} d x+\frac{l}{\delta} \int_{0}^{L} x v_{t}^{2} d x\right) .
$$


By using Young's and Poincaré's inequalities and (22) gives

$$
\begin{aligned}
& -\xi(t) \int_{0}^{L} x u \int_{\tau_{1}}^{\tau_{2}}\left|\mu_{2}(\mathrm{\varrho})\right| z^{2}(x, 1, \mathrm{\varrho}, t) d \mathrm{\varrho} d x \\
& \quad \leq \frac{\xi(t)}{2}\left(C_{p} \delta_{1} \mu_{1} \int_{0}^{L} x u_{x}^{2} d x+\frac{1}{\delta_{1}} \int_{0}^{L} \int_{\tau_{1}}^{\tau_{2}}\left|\mu_{2}(\mathrm{\varrho})\right| z^{2}(x, 1, \mathrm{\varrho}, t) d \mathrm{\varrho} d x\right),
\end{aligned}
$$

$$
\begin{aligned}
& -\xi(t) \int_{0}^{L} x v \int_{\tau_{1}}^{\tau_{2}}\left|\mu_{4}(\mathrm{\varrho})\right| y^{2}(x, 1, \mathrm{\varrho}, t) d \mathrm{\varrho} d x \\
& \quad \leq \frac{\xi(t)}{2}\left(C_{p} \delta_{2} \mu_{3} \int_{0}^{L} x v_{x}^{2} d x+\frac{1}{\delta_{2}} \int_{0}^{L} \int_{\tau_{1}}^{\tau_{2}}\left|\mu_{4}(\mathrm{\varrho})\right| y^{2}(x, 1, \mathrm{\varrho}, t) d \mathrm{\varrho} d x\right) .
\end{aligned}
$$

Similarly, we get

$$
\begin{aligned}
\xi(t) \int_{0}^{L} x u_{t} u d x & \leq \frac{\xi(t)}{2}\left(C_{p} \delta_{1} \mu_{1} \int_{0}^{L} x u_{x}^{2} d x+\frac{\mu_{1}}{\delta_{1}} \int_{0}^{L} x u_{t}^{2} d x\right) \xi(t) \int_{0}^{L} x v_{t} v d x \\
& \leq \frac{\xi(t)}{2}\left(C_{p} \delta_{2} \mu_{3} \int_{0}^{L} x v_{x}^{2} d x+\frac{\mu_{3}}{\delta_{2}} \int_{0}^{L} x v_{t}^{2} d x\right) .
\end{aligned}
$$

In a combination of (98), (99), (100), (101), (102), (103), and (104) in (97), we obtain

$$
\begin{aligned}
\Phi^{\prime}(t) \leq & \left(1+\frac{l}{2 \delta}+\frac{\mu_{1}}{2 \delta_{1}}\right) \xi(t) \int_{0}^{L} x u_{t}^{2} d x \\
& +\left(1+\frac{l}{2 \delta}+\frac{\mu_{3}}{2 \delta_{2}}\right) \xi(t) \int_{0}^{L} x v_{t}^{2} d x \\
& -\frac{\xi(t)}{2}\left[1-\left(1+\eta_{1}\right)\left(1-l_{1}\right)^{2}-\delta C_{p} l-2 \delta_{1} C_{p} \mu_{1}\right] \\
& \cdot \int_{0}^{L} x u_{x}^{2} d x-\frac{\xi(t)}{2}\left[1-\left(1+\eta_{2}\right)\left(1-l_{2}\right)^{2}-\delta C_{p} l-2 \delta_{2} C_{p} \mu_{3}\right] \\
& \cdot \int_{0}^{L} x v_{x}^{2} d x+\frac{\xi(t)}{2}\left(1+\frac{1}{\eta_{1}}\right)\left(\int_{0}^{t} g_{1}^{2-\sigma}(s) d s\right)\left(g_{1}^{\sigma} \circ u_{x}\right)(t) \\
& +\frac{\xi(t)}{2}\left(1+\frac{1}{\eta_{2}}\right)\left(\int_{0}^{t} g_{2}^{2-\sigma}(s) d s\right)\left(g_{2}^{\sigma} \circ v_{x}\right)(t) \\
& +\frac{\xi(t)}{2} \int_{0}^{L} \int_{\tau_{1}}^{\tau_{2}}\left(\frac{1}{\delta_{1}}\left|\mu_{2}(\mathrm{\varrho})\right| z^{2}(x, 1, \varrho, t)\right. \\
& \left.+\frac{1}{\delta_{2}}\left|\mu_{4}(\mathrm{\varrho})\right| y^{2}(x, 1, \mathrm{\varrho}, t)\right) d \mathrm{\varrho} d x \\
& +\frac{\xi(t)}{2(r+2)} \int_{0}^{L}\left[a|u+v|^{2(r+2)}+2 b|u v|^{r+2}\right] d x,
\end{aligned}
$$

by choosing $\eta_{1}, \eta_{1}$, so that $\eta_{1}=l_{1} /\left(1-l_{1}\right)$; hence, $(1 / 2)(-1$ $\left.+\left(1+\eta_{1}\right)\left(1-l_{1}\right)^{2}\right)=-l_{1} / 2$ and $\left(1+\left(1 / \eta_{1}\right)\right)=1 / l_{1}$, and $\eta_{2}=$ $l_{2} /\left(1-l_{2}\right) ; \quad$ therefore, $(1 / 2)\left(-1+\left(1+\eta_{2}\right)\left(1-l_{2}\right)^{2}\right)=-l_{2} / 2$ and $\left(1+\left(1 / \eta_{2}\right)\right)=1 / l_{2}$.

Then, (96) is proved.
Lemma 13. Assuming that $r$ satisfies (15), (14), and (15) and (22) and (52) hold. Then, the functional $\chi(t)$ given by (70) satisfies along the solution of (11)

$$
\begin{aligned}
\chi^{\prime}(t) \leq & \xi(t) \theta\left[1+c_{1}+c_{1}^{\prime}+2\left(1-l_{1}\right)^{2}\right]\left(\int_{0}^{L} x u_{x}^{2} d x\right) \\
& +\xi(t) \theta\left[1+c_{2}+c_{2}^{\prime}+2\left(1-l_{2}\right)^{2}\right]\left(\int_{0}^{L} x v_{x}^{2} d x\right) \\
& +\xi(t)\left[\theta-\left(\int_{0}^{t} g_{1}(s) d s\right)+\theta l+\theta_{1} \mu_{1}\right]\left(\int_{0}^{L} x u_{t}^{2} d x\right) \\
& +\xi(t)\left[\theta-\left(\int_{0}^{t} g_{2}(s) d s\right)+\theta l+\theta_{2} \mu_{3}\right]\left(\int_{0}^{L} x v_{t}^{2} d x\right) \\
+ & {\left[\frac{1}{2 \theta}+2 \theta+\frac{\mu_{1} C_{p}}{2 \theta_{1}}+\frac{C_{p}(1+l)}{4 \theta}\right] } \\
& \times \xi(t)\left(\int_{0}^{t} g_{1}^{2-\sigma}(s) d s\right)\left(g_{1}^{\sigma} \circ u_{x}\right)(t) \\
& +\left[\frac{1}{2 \theta}+2 \theta+\frac{\mu_{3} C_{p}}{2 \theta_{2}}+\frac{C_{p}(1+l)}{4 \theta}\right] \\
& \times \xi(t)\left(\int_{0}^{t} g_{2}^{2-\sigma}(s) d s\right)\left(g_{2}^{\sigma} \circ v_{x}\right)(t) \\
& -\frac{C_{p}}{4 \theta} \xi(t) g_{1}(0)\left(g_{1}^{\prime} \circ u_{x}\right)(t) \\
& -\frac{C_{p}}{4 \theta} \xi(t) g_{2}(0)\left(g_{2}^{\prime} \circ v_{x}\right)(t) \\
+ & \xi(t) \int_{0}^{L} \int_{\tau_{1}}^{\tau_{2}} x\left(\theta_{1}\left|\mu_{2}(\mathrm{\varrho})\right| z^{2}(x, 1, \mathrm{\varrho}, t)\right. \\
+ & \left.\theta_{2}\left|\mu_{4}(\mathrm{\varrho})\right| y^{2}(x, 1, \mathrm{\varrho}, t)\right) d \mathrm{\varrho} d x,
\end{aligned}
$$

for any $\theta, \theta_{1}, \theta_{2}>0$.

Proof. Direct calculation gives

$$
\begin{aligned}
\chi^{\prime}(t)= & -\xi^{\prime}(t) \int_{0}^{L} x u_{t} \int_{0}^{t} g_{1}(t-s)(u(t)-u(s)) d s d x \\
& -\xi(t) \int_{0}^{L} x u_{t t} \int_{0}^{t} g_{1}(t-s)(u(t)-u(s)) d s d x \\
& -\xi(t) \int_{0}^{L} x u_{t} \frac{d}{d t}\left(\int_{0}^{t} g_{1}(t-s)(u(t)-u(s)) d s\right) d x \\
& -\xi^{\prime}(t) \int_{0}^{L} x v_{t} \int_{0}^{t} g_{2}(t-s)(v(t)-v(s)) d s d x \\
& -\xi(t) \int_{0}^{L} x v_{t t} \int_{0}^{t} g_{2}(t-s)(v(t)-v(s)) d s d x \\
& -\xi(t) \int_{0}^{L} x v_{t} \frac{d}{d t}\left(\int_{0}^{t} g_{2}(t-s)(v(t)-v(s)) d s\right) d x
\end{aligned}
$$


by using

$$
\begin{aligned}
\frac{d}{d t}\left(\int_{\alpha(t)}^{\beta(t)} f(t, s) d s\right)= & \int_{\alpha(t)}^{\beta(t)} \frac{\partial f(t, s)}{\partial t} d s \\
& +\frac{\partial \beta(t)}{\partial t} f(t, \beta(t))-\frac{\partial \alpha(t)}{\partial t} f(t, \alpha(t)) .
\end{aligned}
$$

As we have $(u, v, z, y)$ the solution of (11), we find

$$
\begin{aligned}
& \chi^{\prime}(t)=-\xi^{\prime}(t) \int_{0}^{L} x u_{t}\left(\int_{0}^{t} g_{1}(t-s)(u(t)-u(s)) d s\right) d x \\
& +\xi(t) \int_{0}^{L} x u_{x}\left(\int_{0}^{t} g_{1}(t-s)\left(u_{x}(t)-u_{x}(s)\right) d s\right) d x \\
& -\xi(t) \int_{0}^{L} x\left(\int_{0}^{t} g_{1}(t-s) u_{x}(s) d s\right) \\
& \cdot\left(\int_{0}^{t} g_{1}(t-s)\left(u_{x}(t)-u_{x}(s)\right) d s\right) d x \\
& -\xi(t) \mu_{1} \int_{0}^{L} x u_{t}\left(\int_{0}^{t} g_{1}(t-s)(u(t)-u(s)) d s\right) d x \\
& -\xi(t) \int_{0}^{L} x\left(\int_{\tau_{1}}^{\tau_{2}}\left|\mu_{2}(\rho)\right| z^{2}(x, 1, \rho, t) d \rho\right) \\
& \cdot\left(\int_{0}^{t} g_{1}(t-s)(u(t)-u(s)) d s\right) d x-\xi(t) \int_{0}^{L} x f_{1}(u, v) \\
& \cdot\left(\int_{0}^{t} g_{1}(t-s)(u(t)-u(s)) d s\right) d x-\xi(t) \int_{0}^{L} x u_{t} \\
& \cdot\left(\int_{0}^{t} g_{1}^{\prime}(t-s)(u(t)-u(s)) d s\right) d x-\xi(t) \\
& \cdot\left(\int_{0}^{t} g_{1}(s) d s\right) \int_{0}^{L} x u_{t}^{2} d x-\xi(t)\left(\int_{0}^{t} g_{2}(s) d s\right) \int_{0}^{L} x v_{t}^{2} d x \\
& -\xi^{\prime}(t) \int_{0}^{L} x v_{t}\left(\int_{0}^{t} g_{2}(t-s)(v(t)-v(s)) d s\right) d x \\
& +\xi(t) \int_{0}^{L} x v_{x}\left(\int_{0}^{t} g_{2}(t-s)\left(v_{x}(t)-v_{x}(s)\right) d s\right) d x \\
& -\xi(t) \int_{0}^{L} x\left(\int_{0}^{t} g_{2}(t-s) v_{x}(s) d s\right) \\
& \cdot\left(\int_{0}^{t} g_{2}(t-s)\left(v_{x}(t)-v_{x}(s)\right) d s\right) d x-\xi(t) \mu_{3} \int_{0}^{L} x v_{t} \\
& \left(\int_{0}^{t} g_{2}(t-s)(v(t)-v(s)) d s\right) d x-\xi(t) \int_{0}^{L} x \\
& \left(\int_{\tau_{1}}^{\tau_{2}}\left|\mu_{4}(\rho)\right| y^{2}(x, 1, \rho, t) d \rho\right) \\
& \cdot\left(\int_{0}^{t} g_{2}(t-s)(v(t)-v(s)) d s\right) d x \\
& -\xi(t) \int_{0}^{L} x f_{2}(u, v)\left(\int_{0}^{t} g_{2}(t-s)(v(t)-v(s)) d s\right) d x \\
& -\xi(t) \int_{0}^{L} x v_{t}\left(\int_{0}^{t} g_{2}^{\prime}(t-s)(v(t)-v(s)) d s\right) d x \text {. }
\end{aligned}
$$

By Young's inequality and (14) and (15), we arrive to

$$
\begin{aligned}
& -\xi^{\prime}(t) \int_{0}^{L} x u_{t}\left(\int_{0}^{t} g_{1}(t-s)(u(t)-u(s)) d s\right) d x \\
& \leq \xi(t)\left|\frac{\xi^{\prime}(t)}{\xi(t)}\right|\left[\theta \int_{0}^{L} x u_{t}^{2} d x+\frac{C_{p}}{4 \theta}\left(\int_{0}^{t} g_{1}^{2-\sigma}(s) d s\right)\left(g_{1}^{\sigma} \circ u_{x}\right)(t)\right] \\
& \leq \theta l \xi(t) \int_{0}^{L} x u_{t}^{2} d x+\frac{C_{p} l}{4 \theta} \xi(t)\left(\int_{0}^{t} g_{1}^{2-\sigma}(s) d s\right)\left(g_{1}^{\sigma} \circ u_{x}\right)(t), \\
& \xi(t) \int_{0}^{L} x u_{x}\left(\int_{0}^{t} g_{1}(t-s)\left(u_{x}(t)-u_{x}(s)\right) d s\right) d x \\
& \leq \theta \xi(t) \int_{0}^{L} x u_{x}^{2} d x+\frac{1}{4 \theta} \xi(t)\left(\int_{0}^{t} g_{1}^{2-\sigma}(s) d s\right)\left(g_{1}^{\sigma} \circ u_{x}\right)(t) .
\end{aligned}
$$

\section{Similarly, we get}

$$
\begin{aligned}
& \xi(t) \mu_{1} \int_{0}^{L} x u_{t}\left(\int_{0}^{t} g_{1}(t-s)(u(t)-u(s)) d s\right) d x \\
& \leq \theta_{1} \mu_{1} \xi(t) \int_{0}^{L} x u_{t}^{2} d x+\frac{1}{4 \theta_{1}} C_{p} \xi(t)\left(\int_{0}^{t} g_{1}^{2-\sigma}(s) d s\right)\left(g_{1}^{\sigma} \circ u_{x}\right)(t) \\
& \xi(t) \mu_{3} \int_{0}^{L} x v_{t}\left(\int_{0}^{t} g_{2}(t-s)(v(t)-v(s)) d s\right) d x \\
& \leq \theta_{2} \mu_{3} \xi(t) \int_{0}^{L} x v_{t}^{2} d x+\frac{1}{4 \theta_{2}} C_{p} \xi(t)\left(\int_{0}^{t} g_{2}^{2-\sigma}(s) d s\right)\left(g_{2}^{\sigma} \circ v_{x}\right)(t)
\end{aligned}
$$

with

$$
\begin{aligned}
& -\xi(t) \int_{0}^{L} x\left(\int_{0}^{t} g_{1}(t-s) u_{x}(s) d s\right)\left(\int_{0}^{t} g_{1}(t-s)\left(u_{x}(t)-u_{x}(s)\right) d s\right) d x \\
& \leq 2 \theta\left(1-l_{1}\right)^{2} \xi(t) \int_{0}^{L} x u_{x}^{2} d x+\left(2 \theta+\frac{1}{4 \theta}\right) \xi(t) \\
& \cdot\left(\int_{0}^{t} g_{1}^{2-\sigma}(s) d s\right)\left(g_{1}^{\sigma} \circ u_{x}\right)(t) .
\end{aligned}
$$

So

$$
\begin{aligned}
& -\xi(t) \int_{0}^{L} x f_{1}(u, v)\left(\int_{0}^{t} g_{1}(t-s)(u(t)-u(s)) d s\right) d x \\
& \leq \frac{C_{p}}{4 \theta} \xi(t)\left(\int_{0}^{t} g_{1}^{2-\sigma}(s) d s\right)\left(g_{1}^{\sigma} \circ u_{x}\right)(t) \\
& \quad+c_{1} \theta \xi(t) \int_{0}^{L} x u_{x}^{2} d x+c_{2} \theta \xi(t) \int_{0}^{L} x v_{x}^{2} d x
\end{aligned}
$$


where

$$
\begin{aligned}
& \left\{\begin{array}{l}
c_{1}:=\Lambda_{1}\left(\frac{2(r+2)}{(r+1)} E(0)\right)^{2(r+1)}, \\
c_{2}:=\Lambda_{2}\left(\frac{2(r+2)}{(r+1)} E(0)\right)^{2(r+1)},
\end{array}\right. \\
& -\xi(t) \int_{0}^{L} x u_{t}\left(\int_{0}^{t} g_{1}^{\prime}(t-s)(u(t)-u(s)) d s\right) d x \\
& \leq \theta \xi(t) \int_{0}^{L} x u_{t}^{2} d x-\frac{g_{1}(0)}{4 \theta} C_{p} \xi(t)\left(g_{1}^{\prime} \circ u_{x}\right)(t) \text {. }
\end{aligned}
$$

Then,

$$
\begin{aligned}
& -\xi^{\prime}(t) \int_{0}^{L} x v_{t}\left(\int_{0}^{t} g_{2}(t-s)(v(t)-v(s)) d s\right) d x \\
& \leq \theta l \xi(t) \int_{0}^{L} x v_{t}^{2} d x+\frac{C_{p} l}{4 \theta} \xi(t)\left(\int_{0}^{t} g_{2}^{2-\sigma}(s) d s\right)\left(g_{2}^{\sigma} \circ v_{x}\right)(t) \\
& \xi(t) \int_{0}^{L} x v_{x}\left(\int_{0}^{t} g_{2}(t-s)\left(v_{x}(t)-v_{x}(s)\right) d s\right) d x \\
& \leq \theta \xi(t) \int_{0}^{L} x v_{x}^{2} d x+\frac{1}{4 \theta} \xi(t)\left(\int_{0}^{t} g_{2}^{2-\sigma}(s) d s\right)\left(g_{2}^{\sigma} \circ v_{x}\right)(t)
\end{aligned}
$$

Thus,

$$
\begin{aligned}
& -\xi(t) \int_{0}^{L} x\left(\int_{0}^{t} g_{2}(t-s) v_{x}(s) d s\right)\left(\int_{0}^{t} g_{2}(t-s)\left(v_{x}(t)-v_{x}(s)\right) d s\right) d x \\
& \leq 2 \theta\left(1-l_{2}\right)^{2} \xi(t) \int_{0}^{L} x v_{x}^{2} d x+\left(2 \theta+\frac{1}{4 \theta}\right) \xi(t) \\
& \cdot\left(\int_{0}^{t} g_{2}^{2-\sigma}(s) d s\right)\left(g_{2}^{\sigma} \circ v_{x}\right)(t) \\
& -\frac{\xi(t)}{2(r+2)} \int_{0}^{L} x f_{2}(u, v)\left(\int_{0}^{t} g_{2}(t-s)(v(t)-v(s)) d s\right) d x \\
& \leq \frac{C_{p}}{4 \theta} \xi(t)\left(\int_{0}^{t} g_{2}^{2-\sigma}(s) d s\right)\left(g_{2}^{\sigma} \circ v_{x}\right)(t)+c_{1}^{\prime} \theta \xi(t) \\
& \cdot \int_{0}^{L} x u_{x}^{2} d x+c_{2}^{\prime} \theta \xi(t) \int_{0}^{L} x v_{x}^{2} d x
\end{aligned}
$$

where

$$
\left\{\begin{array}{l}
c_{1}^{\prime}:=\Lambda_{1}^{\prime}\left(\frac{2(r+2)}{r+1} E(0)\right)^{2(r+1)} \\
c_{2}^{\prime}:=\Lambda_{2}^{\prime}\left(\frac{2(r+2)}{r+1} E(0)\right)^{2(r+1)}
\end{array}\right.
$$

$$
\begin{aligned}
& -\xi(t) \int_{0}^{L} x v_{t}\left(\int_{0}^{t} g_{2}^{\prime}(t-s)(v(t)-v(s)) d s\right) d x \\
& \quad \leq \theta \xi(t) \int_{0}^{L} x v_{t}^{2} d x-\frac{g_{2}(0)}{4 \theta} C_{p} \xi(t)\left(g_{2}^{\prime} \circ v_{x}\right)(t) .
\end{aligned}
$$

Similarly, we have

$$
\begin{gathered}
-\xi(t) \int_{0}^{L} x\left(\int_{\tau_{1}}^{\tau_{2}}\left|\mu_{2}(\rho)\right| z^{2}(x, 1, \rho, t) d \rho\right) \\
\cdot\left(\int_{0}^{t} g_{1}(t-s)(u(t)-u(s)) d s\right) d x \theta_{1} \xi(t) \\
\cdot \int_{0}^{L} \int_{\tau_{1}}^{\tau_{2}} x\left|\mu_{2}(\rho)\right| z^{2}(x, 1, \rho, t) d \rho d x \\
+\frac{1}{4 \theta_{1}} \mu_{1} C_{p}\left(\int_{0}^{t} g_{1}^{2-\sigma}(s) d s\right)\left(g_{1}^{\sigma} \circ u_{x}\right)(t)
\end{gathered}
$$

A combination of (110), (111), (112), (113), (114), (115), (117), (118), (119), (120), (121), (123), (124), and (125) into (109) gives (106).

Lemma 14. Let $(u, v, z, y)$ be the solution of (11). Then, for $\eta_{3}>0$, the functional $\Psi(t)$ satisfies

$$
\begin{aligned}
\Psi^{\prime}(t) \leq & -\xi(t) \eta_{4} \int_{0}^{L} \int_{0}^{1} \int_{\tau_{1}}^{\tau_{2}} x \mathrm{\varrho}\left(\left|\mu_{2}(\mathrm{\varrho})\right| z^{2}+\left|\mu_{4}(\mathrm{\varrho})\right| y^{2}\right) d \mathrm{\varrho} d \rho d x \\
& +\xi(t) \mu_{1} \int_{0}^{L} x u_{t}^{2} d x+\xi(t) \mu_{3} \int_{0}^{L} x v_{t}^{2} d x \\
& -\xi(t) \eta_{3} \int_{0}^{L} \int_{\tau_{1}}^{\tau_{2}} x\left(\left|\mu_{2}(\mathrm{\varrho})\right| z^{2}(x, 1, \mathrm{\varrho}, t)\right. \\
& \left.+\left|\mu_{4}(\mathrm{\varrho})\right| y^{2}(x, 1, \mathrm{\varrho}, t)\right) d \mathrm{\varrho} d x
\end{aligned}
$$

where $\eta_{3}>0$ and $\eta_{4}=\eta_{3}(1-l)>0>0$. 
Proof. By differentiating $\Psi(t)$ and using equations $(11)_{3}$ and $(11)_{4}$, we get

$$
\begin{aligned}
\Psi^{\prime}(t)= & \xi^{\prime}(t) \int_{0}^{L} \int_{0}^{1} \int_{\tau_{1}}^{\tau_{2}} x \rho e^{-\mathrm{\varrho} \rho}\left(\left|\mu_{2}(\mathrm{\varrho})\right| z^{2}+\left|\mu_{4}(\mathrm{\varrho})\right| z^{2}\right) d \varrho d \rho d x \\
& -2 \xi(t) \int_{0}^{L} \int_{0}^{1} \int_{\tau_{1}}^{\tau_{2}} e^{-\mathrm{\varrho} \rho}\left|\mu_{2}(\mathrm{\varrho})\right| z z_{\rho}(x, \rho, \varrho, t) d \varrho d \rho d x \\
& -2 \xi(t) \int_{0}^{L} \int_{0}^{1} \int_{\tau_{1}}^{\tau_{2}} e^{-\mathrm{\varrho} \rho}\left|\mu_{4}(\mathrm{\varrho})\right| y y_{\rho}(x, \rho, \varrho, t) d \varrho d \rho d x \\
= & \xi^{\prime}(t) \int_{0}^{L} \int_{0}^{1} \int_{\tau_{1}}^{\tau_{2}} x \mathrm{\varrho} e^{-\mathrm{\varrho} \rho}\left(\left|\mu_{2}(\mathrm{\varrho})\right| z^{2}+\left|\mu_{4}(\mathrm{\varrho})\right| z^{2}\right) d \mathrm{\varrho} d \rho d x \\
& -\xi(t) \int_{0}^{L} \int_{0}^{1} \int_{\tau_{1}}^{\tau_{2}} x \mathrm{\varrho} e^{-\mathrm{\varrho} \rho}\left|\mu_{2}(\mathrm{\varrho})\right| z^{2} d \mathrm{\varrho} d \rho d x \\
& -\xi(t) \int_{0}^{L} \int_{\tau_{1}}^{\tau_{2}} x\left|\mu_{2}(\mathrm{\varrho})\right|\left[e^{-\mathrm{\varrho}} z^{2}(x, 1, \mathrm{\varrho}, t)-z^{2}(x, 0, \varrho, t)\right] d \mathrm{\varrho} d x \\
& -\xi(t) \int_{0}^{L} \int_{0}^{1} \int_{\tau_{1}}^{\tau_{2}} x \mathrm{\varrho} e^{-\mathrm{\varrho} \rho}\left|\mu_{4}(p)\right| y^{2} d \rho \mathrm{\varrho} \rho d x \\
& -\xi(t) \int_{0}^{L} \int_{\tau_{1}}^{\tau_{2}} x\left|\mu_{4}(\mathrm{\varrho})\right|\left[e^{-\mathrm{\varrho}} y^{2}(x, 1, \varrho, t)-y^{2}(x, 0, \varrho, t)\right] d \varrho d x .
\end{aligned}
$$

Using the equality $z(x, 0, \varrho, t)=u_{t}(x, t), y(x, 0, \varrho, t)=$ $v_{t}(x, t)$, and $e^{-\mathrm{e}} \leq e^{-\rho \mathrm{Q}} \leq 1$, for any $0<\rho<1$, we find

$$
\begin{aligned}
\Psi^{\prime}(t) \leq & \xi(t) l \int_{0}^{L} \int_{0}^{1} \int_{\tau_{1}}^{\tau_{2}} x \mathrm{\varrho}\left(\left|\mu_{2}(\mathrm{\varrho})\right| z^{2}+\left|\mu_{4}(\mathrm{\varrho})\right| z^{2}\right) d \mathrm{\varrho} d \rho d x \\
& -\xi(t) \int_{0}^{L} \int_{0}^{1} \int_{\tau_{1}}^{\tau_{2}} x \varrho e^{-\mathrm{\varrho} \rho}\left(\left|\mu_{2}(\mathrm{\varrho})\right| z^{2}+\left|\mu_{4}(\mathrm{\varrho})\right| y^{2}\right) d \mathrm{\varrho} d \rho d x \\
& -\xi(t) \int_{0}^{L} \int_{\tau_{1}}^{\tau_{2}} x e^{-\varrho}\left(\left|\mu_{2}(\mathrm{\varrho})\right| z^{2}(x, 1, \mathrm{\varrho}, t)+\left|\mu_{4}(\mathrm{\varrho})\right| y^{2}(x, 1, \mathrm{\varrho}, t)\right) d \mathrm{\varrho} d x \\
& +\left(\int_{\tau_{1}}^{\tau_{2}}\left|\mu_{2}(\mathrm{\varrho})\right| d \mathrm{\varrho}\right) \xi(t) \int_{0}^{L} x u_{t}^{2} d x \\
& +\left(\int_{\tau_{1}}^{\tau_{2}}\left|\mu_{4}(\mathrm{\varrho})\right| d \mathrm{\varrho}\right) \xi(t) \int_{0}^{L} x v_{t}^{2} d x .
\end{aligned}
$$

As $-e^{-\varrho}$ is an increasing function, we have $-e^{-\varrho} \leq-e^{-\tau_{2}}$, for any $\rho \in\left[\tau_{1}, \tau_{2}\right]$.

Then, setting $\eta_{3}=e^{-\tau_{2}}$ and (22), we obtain (126).

Theorem 15. Let $\left(u_{0}, v_{0}\right) \in V_{0}^{2},\left(u_{1}, v_{1}\right) \in H^{2}$, and $\left(f_{0}, g_{0}\right) \in$ $L_{x}^{2}\left((0, L) \times(0,1) \times\left(\tau_{1}, \tau_{2}\right)\right)$ be defined and satisfy (163). Assume that $r$ satisfies (24), (14), (15), (16), (17), and (22) hold. Then, for each $t_{0}>0, \exists K$ and $k$ such that the solution of (11) satisfies $\forall t \geq t_{0}$, we have the following inequality for the energy function

$$
E(t) \leq \begin{cases}K e^{-k \int_{t_{0}}^{t} \xi(s) d s}, & \sigma=1, \\ K\left(1+\int_{t_{0}}^{t} \xi(s) d s\right)^{-(1 /(\sigma-1))}, & 1<\sigma<\frac{3}{2} .\end{cases}
$$

Proof. As $g_{1}, g_{2}$ is continuous and $g_{1}(0), g_{2}(0)>0$, hence $\forall$ $t_{0}>0$; we have

$$
\begin{cases}\int_{0}^{t} g_{1}(s) d s \geq \int_{t_{0}}^{t} g_{1}(s) d s=g_{1,0}>0, & \forall t \geq t_{0}, \\ \int_{0}^{t} g_{2}(s) d s \geq \int_{t_{0}}^{t} g_{2}(s) d s=g_{2,0}>0, & \forall t \geq t_{0} .\end{cases}
$$

By using (36), (96), (106), (126), and (130) and $0<\xi(t)$ $\leq \xi(0))$ (hence $(\xi(t) / \xi(0))<1)$, we get

$$
\begin{aligned}
& F^{\prime}(t)=E^{\prime}(t)+\varepsilon_{1} \Phi^{\prime}(t)+\varepsilon_{2} \chi^{\prime}(t)+\varepsilon_{3} \Psi^{\prime}(t) \\
& \leq-\left[d_{1}-\varepsilon_{1}\left(1+\frac{1}{2 \delta}+\frac{\mu_{1}}{2 \delta_{1}}\right)\right. \\
& \left.+\varepsilon_{2}\left(g_{1,0}-\theta-\theta l-\mu_{1} \theta_{1}\right)-\varepsilon_{3} \mu_{1}\right] \xi(t)\left(\int_{0}^{L} x u_{t}^{2} d x\right) \\
& -\left[d_{2}-\varepsilon_{1}\left(1+\frac{1}{2 \delta}+\frac{\mu_{3}}{2 \delta_{2}}\right)+\varepsilon_{2}\left(g_{2,0}-\theta-\theta l-\mu_{1} \theta_{1}\right)\right. \\
& \left.-\varepsilon_{3} \mu_{3}\right] \xi(t)\left(\int_{0}^{L} x v_{t}^{2} d x\right)+2 \varepsilon_{1} \xi(t) \\
& \cdot \int_{0}^{L} x\left[a|u+v|^{2(r+2)}+2 b|u v|^{r+2}\right] d x \\
& +\left(\frac{1}{2}-\frac{\varepsilon_{2} \xi(0)}{4 \theta} C_{p} g_{1}(0)\right)\left(g_{1}^{\prime} \circ u_{x}\right)(t) \\
& +\left(\frac{1}{2}-\frac{\varepsilon_{2} \xi(0)}{4 \theta} C_{p} g_{2}(0)\right)\left(g_{2}^{\prime} \circ v_{x}\right)(t) \\
& \text { - }\left[\frac{\varepsilon_{1}}{2}\left(l_{1}-\delta C_{p} l-2 \delta_{1} \mu_{1} C_{p}\right)-\varepsilon_{2} \theta\right. \\
& \left.\cdot\left(1+c_{1}+c_{1}^{\prime}+2\left(1-l_{1}\right)^{2}\right)\right] \xi(t) \\
& \left(\int_{0}^{L} x u_{x}^{2} d x\right)-\left[\frac{\varepsilon_{1}}{2}\left(l_{2}-\delta C_{p} l-2 \delta_{2} \mu_{3} C_{p}\right)\right. \\
& \left.-\varepsilon_{2} \theta\left(1+c_{2}+c_{2}^{\prime}+2\left(1-l_{2}\right)^{2}\right)\right] \xi(t)\left(\int_{0}^{L} x v_{x}^{2} d x\right) \\
& +\left[\frac{\varepsilon_{1}}{2 l_{1}}+\varepsilon_{2}\left(\frac{1}{2 \theta}+2 \theta+\frac{\mu_{1} C_{p}}{2 \theta_{1}}+\frac{C_{p}+l C_{p}}{4 \theta}\right)\right] \xi(t) \\
& \cdot\left(\int_{0}^{t} g_{1}^{2-\sigma}(s) d s\right)\left(g_{1}^{\sigma} \circ u_{x}\right)(t) \\
& +\left[\frac{\varepsilon_{1}}{2 l_{2}}+\varepsilon_{2}\left(\frac{1}{2 \theta}+2 \theta+\frac{\mu_{1} C_{p}}{2 \theta_{1}}+\frac{C_{p}+l C_{p}}{4 \theta}\right)\right] \xi(t) \\
& \cdot\left(\int_{0}^{t} g_{2}^{2-\sigma}(s) d s\right)\left(g_{2}^{\sigma} \circ v_{x}\right)(t) \\
& -\left[\varepsilon_{3} \eta_{3}-\varepsilon_{1} \frac{1}{2 \delta_{1}}-\varepsilon_{2} \theta_{1}\right] \xi(t) \\
& \int_{0}^{L} \int_{\tau_{1}}^{\tau_{2}} x\left|\mu_{2}(\varrho)\right| z^{2}(x, 1, \varrho, t) d \varrho d x \\
& -\left[\varepsilon_{3} \eta_{3}-\varepsilon_{1} \frac{1}{2 \delta_{2}}-\varepsilon_{2} \theta_{2}\right] \xi(t) \\
& \cdot \int_{0}^{L} \int_{\tau_{1}}^{\tau_{2}} x\left|\mu_{4}(\mathrm{\varrho})\right| y^{2}(x, 1, \mathrm{\varrho}, t) d \mathrm{\varrho} d x \\
& -\varepsilon_{3} \eta_{4} \xi(t) \int_{0}^{L} \int_{0}^{1} \int_{\tau_{1}}^{\tau_{2}} x \rho\left(\left|\mu_{2}(\mathrm{\varrho})\right| z^{2}+\left|\mu_{4}(\mathrm{\varrho})\right| y^{2}\right) d \mathrm{\varrho} d \rho d x .
\end{aligned}
$$


By choosing $\delta, \delta_{1}$, and $\delta_{2}$ so small that

$$
\left\{\begin{array}{l}
\left(l_{1}-\delta C_{p} l-2 \mu_{1} \delta_{1} C_{p}\right)>\frac{l_{1}}{2}, \\
\left(l_{2}-\delta C_{p} l-2 \mu_{3} \delta_{2} C_{p}\right)>\frac{l_{2}}{2} .
\end{array}\right.
$$

Then,

$$
\begin{gathered}
\delta<\frac{1}{4 C_{p} l} \min \left\{l_{1}, l_{2}\right\}, \\
\delta_{1}<\frac{1}{8 \mu_{1} C_{p}} \min \left\{l_{1}, l_{2}\right\}, \\
\delta_{2}<\frac{1}{8 \mu_{3} C_{p}} \min \left\{l_{1}, l_{2}\right\} .
\end{gathered}
$$

At this point, we choose $\theta$ small enough, such that

$$
\begin{aligned}
& k_{3}:=\frac{\varepsilon_{1} l_{1}}{4}-\varepsilon_{2} \theta\left(1+c_{1}+c_{1}^{\prime}+2\left(1-l_{1}\right)^{2}\right)>0, \\
& k_{4}:=\frac{\varepsilon_{1} l_{2}}{4}-\varepsilon_{2} \theta\left(1+c_{2}+c_{2}^{\prime}+2\left(1-l_{2}\right)^{2}\right)>0 .
\end{aligned}
$$

Then,

$$
\theta<\min \left\{\frac{\varepsilon_{1} l_{1}}{4 \varepsilon_{2}\left(1+c_{1}+c_{1}^{\prime}+2\left(1-l_{1}\right)^{2}\right)}, \frac{\varepsilon_{1} l_{2}}{4 \varepsilon_{2}\left(1+c_{2}+c_{2}^{\prime}+2\left(1-l_{2}\right)^{2}\right)}\right\} .
$$

Now, $\delta, \delta_{1}, \delta_{2}$, and $\theta$ are fixed. Then, we select $\varepsilon_{1}, \varepsilon_{2}, \varepsilon_{3}$, $\theta_{1}$, and $\theta_{2}$ so small that (72) and (162) remain correct and

$$
\begin{aligned}
& k_{1}:=\left[d_{1}-\varepsilon_{1}\left(1+\frac{1}{2 \delta}+\frac{\mu_{1}}{2 \delta_{1}}\right)+\varepsilon_{2}\left(g_{1,0}-\mu_{1} \theta_{1}-(1+l) \theta\right)-\varepsilon_{3} \mu_{1}\right]>0, \\
& k_{2}:=\left[d_{2}-\varepsilon_{1}\left(1+\frac{1}{2 \delta}+\frac{\mu_{3}}{2 \delta_{2}}\right)+\varepsilon_{2}\left(g_{2,0}-\mu_{3} \theta_{2}-(1+l) \theta\right)-\varepsilon_{3} \mu_{3}\right]>0, \\
& k_{5}:=\left(\frac{1}{2}-\frac{\varepsilon_{2} \xi(0)}{4 \theta} C_{p} g_{1}(0)\right)-\left\{\left[\frac{\varepsilon_{1}}{2 l_{1}}+\varepsilon_{2}\left(\frac{1}{2 \theta}+2 \theta+\frac{\mu_{1} C_{p}}{2 \theta_{1}}+\frac{C_{p}+l C_{p}}{4 \theta}\right)\right]\left(\int_{0}^{t} g_{1}^{2-\sigma}(s) d s\right)\right\}>0, \\
& k_{6}:=\left(\frac{1}{2}-\frac{\varepsilon_{2} \xi(0)}{4 \theta} C_{p} g_{2}(0)\right)-\left\{\left[\frac{\varepsilon_{1}}{2 l_{2}}+\varepsilon_{2}\left(\frac{1}{2 \theta}+2 \theta+\frac{\mu_{3} C_{p}}{2 \theta_{2}}+\frac{C_{p}+l C_{p}}{4 \theta}\right)\right]\left(\int_{0}^{t} g_{2}^{2-\sigma}(s) d s\right)\right\}>0, \\
& k_{7}:=\varepsilon_{3} \eta_{3}-\varepsilon_{1} \frac{1}{2 \delta_{1}}-\varepsilon_{2} \theta_{1}>0, \\
& k_{8}:=\varepsilon_{3} \eta_{3}-\varepsilon_{1} \frac{1}{2 \delta_{2}}-\varepsilon_{2} \theta_{2}>0 .
\end{aligned}
$$

Hence, by using (15) gives, for some $\sigma>0$,

$$
\begin{aligned}
F^{\prime}(\mathrm{t}) \leq & -\sigma \xi(t)\left[\int_{0}^{L} x u_{t}^{2} d x+\int_{0}^{L} x v_{t}^{2} d x\right. \\
& -\int_{0}^{L} x\left[a|u+v|^{2(r+2)}+2 b|u v|^{r+2}\right] d x \\
& +\int_{0}^{L} x u_{x}^{2} d x+\int_{0}^{L} x v_{x}^{2} d x+\left(g_{1}^{\sigma} \circ u_{x}\right)(t) \\
& \left.+\left(g_{2}^{\sigma} \circ v_{x}\right)(t)+K(z, y)\right] .
\end{aligned}
$$

We choose $\theta, \theta_{1}$, and $\theta_{2}$ so small that

$$
\begin{aligned}
& \left(g_{1,0}-\mu_{1} \theta_{1}-(1+l) \theta\right)>\frac{1}{2} g_{1,0}, \\
& \left(g_{2,0}-\mu_{3} \theta_{2}-(1+l) \theta\right)>\frac{1}{2} g_{2,0} .
\end{aligned}
$$

By (134), we get

$$
\begin{aligned}
& \theta<\min \left\{\frac{\varepsilon_{1} l_{1}}{4 \varepsilon_{2}\left(1+c_{1}+c_{1}^{\prime}+2\left(1-l_{1}\right)^{2}\right)}, \frac{\varepsilon_{1} l_{2}}{4 \varepsilon_{2}\left(1+c_{2}+c_{2}^{\prime}+2\left(1-l_{2}\right)^{2}\right)},\right. \\
& \theta_{1}<\frac{1}{4 \mu_{1}(1+l)} g_{1,0}, \theta_{2}<\frac{1}{4 \mu_{3}(1+l)} g_{2,0}, \\
& \quad \frac{4 \theta\left(1+c_{1}+c_{1}^{\prime}+2\left(1-l_{1}\right)^{2}\right)}{l_{1}}<\frac{1}{2+(1 / \delta)+\left(\mu_{1} / \delta_{1}\right)}, \\
& \frac{4 \theta\left(1+c_{2}+c_{2}^{\prime}+2\left(1-l_{2}\right)^{2}\right)}{l_{2}}<\frac{g_{2,0}}{2+(l / \delta)+\left(\mu_{3} / \delta_{2}\right)} .
\end{aligned}
$$


With $\theta, \theta_{1}, \theta_{2}$, and $\alpha^{\prime}$ fixed, we pick $\varepsilon_{1}, \varepsilon_{2}$, and $\varepsilon_{3}$ such that

$$
\begin{aligned}
& \max \left\{\frac{4 \theta\left(1+c_{1}+c_{1}^{\prime}+2\left(1-l_{1}\right)^{2}\right)}{l_{1}}, \frac{4 \theta\left(1+c_{2}+c_{2}^{\prime}+2\left(1-l_{2}\right)^{2}\right)}{l_{2}}\right\} \varepsilon_{2} \\
& <\varepsilon_{1}<\frac{1}{2+(l / \delta)+\min \left(\left(\mu_{1} / \delta_{1}\right),\left(\mu_{3} / \delta_{2}\right)\right)}\left(\min \left(d_{1}, d_{2}\right)\right. \\
& \left.\quad+\varepsilon_{2} \min \left\{g_{1,0} ’_{2,0}\right\}+\varepsilon_{3} \min \left(\mu_{1}+\mu_{3}\right)\right) .
\end{aligned}
$$

We will make

$$
\left\{\begin{array}{l}
k_{1}:=\left[d_{1}-\varepsilon_{1}\left(1+\frac{1}{2 \delta}+\frac{\mu_{1}}{2 \delta_{1}}\right)+\varepsilon_{2}\left(g_{1,0}-\mu_{1} \theta_{1}-(1+l) \theta\right)-\varepsilon_{3} \mu_{1}\right]>0, \\
k_{2}:=\left[-\varepsilon_{1}\left(1+\frac{1}{2 \delta}+\frac{\mu_{3}}{2 \delta_{2}}\right)+\varepsilon_{2}\left(g_{2,0}-\mu_{3} \theta_{2}-\theta-\theta l\right)-\varepsilon_{3} \mu_{3}\right]>0, \\
k_{3}:=\frac{\varepsilon_{1}}{2}\left(l_{1}-2 \mu_{1} \delta_{1} C_{p}-\delta C_{p} l\right)-\varepsilon_{2} \theta\left(1+c_{1}+c_{1}^{\prime}+2\left(1-l_{1}\right)^{2}\right)>0, \\
k_{4}:=\frac{\varepsilon_{1}}{2}\left(l_{2}-2 \mu_{3} \delta_{2} C_{p}-\delta C_{p} l\right)-\varepsilon_{2} \theta\left(1+c_{2}+c_{2}^{\prime}+2\left(1-l_{2}\right)^{2}\right)>0, \\
k_{7}:=\varepsilon_{3} \eta_{3}-\varepsilon_{1} \frac{1}{2 \delta_{1}}-\varepsilon_{2} \theta_{1}>0, \\
k_{8}:=\varepsilon_{3} \eta_{3}-\varepsilon_{1} \frac{1}{2 \delta_{2}}-\varepsilon_{2} \theta_{2}>0 .
\end{array}\right.
$$

Then, we select $\varepsilon_{1}, \varepsilon_{2}$, and $\varepsilon_{3}$ so small that (72) and (137) remain correct and

$$
\begin{aligned}
k_{5}:= & \left(\frac{1}{2}-\frac{\varepsilon_{2} \xi(0)}{4 \theta} C_{p} g_{1}(0)\right)-\left\{\left[\frac{\varepsilon_{1}}{2 l_{1}}+\varepsilon_{2}\left(\frac{1}{2 \theta}+2 \theta+\frac{\mu_{1} C_{p}}{2 \theta_{1}}\right.\right.\right. \\
& \left.\left.\left.+\frac{C_{p}+l C_{p}}{4 \theta}\right)\right]\left(\int_{0}^{t} g_{1}^{2-\sigma}(s) d s\right)\right\}>0, \\
k_{6}:= & \left(\frac{1}{2}-\frac{\varepsilon_{2} \xi(0)}{4 \theta} C_{p} g_{2}(0)\right)-\left\{\left[\frac{\varepsilon_{1}}{2 l_{2}}+\varepsilon_{2}\left(\frac{1}{2 \theta}+2 \theta+\frac{\mu_{3} C_{p}}{2 \theta_{2}}\right.\right.\right. \\
& \left.\left.\left.+\frac{C_{p}+l C_{p}}{4 \theta}\right)\right]\left(\int_{0}^{t} g_{2}^{2-\sigma}(s) d s\right)\right\}>0 .
\end{aligned}
$$

Next, as (137) is showed, according to the different ranges of $r$, we give the following two cases.

Case 1. $\sigma=1$.

By choosing $\varepsilon_{1}, \varepsilon_{2}, \varepsilon_{3}, \theta_{1}, \theta_{2}$, and $\theta$, (137) gives, for $\gamma>0$ is constant so that,

$$
F^{\prime}(t) \leq-\gamma \xi(t) E(t), \quad \forall t \geq t_{0}
$$

Therefore, with the help of the LHS of (72) and (143), we obtain

$$
F^{\prime}(t) \leq-\gamma \alpha_{1} \xi(t) F(t), \forall t \geq t_{0}
$$

By integration of (144) over $\left(t_{0}, t\right)$ gives

$$
F^{\prime}(t) \leq F\left(t_{0}\right) e^{\left(-\gamma \alpha_{1}\right) \int_{t_{0}}^{t} \xi(s) d s}, \quad \forall t \geq t_{0}
$$

Therefore, $(129)_{1}$ is proved by (72) as well.

Case 2. $1<\sigma<3 / 2$.

We use (11), which gives

$$
\begin{aligned}
& g_{1}(t)^{1-\sigma} \geq(\sigma-1)\left(\int_{t_{0}}^{t} \xi(s) d s\right)+g_{1}\left(t_{0}\right)^{1-\sigma}, \\
& g_{2}(t)^{1-\sigma} \geq(\sigma-1)\left(\int_{t_{0}}^{t} \xi(s) d s\right)+g_{2}\left(t_{0}\right)^{1-\sigma} .
\end{aligned}
$$

We have, for $0<\tau<1$,

$$
\begin{aligned}
& \int_{0}^{\infty} g_{1}^{1-\tau}(s) d s \leq \int_{0}^{\infty} \frac{1}{\left[(\sigma-1)\left(\int_{t_{0}}^{t} \xi(s) d s\right)+g_{1}\left(t_{0}\right)^{1-\sigma}\right]^{(1-\tau) /(\sigma-1)}} d s \\
& \int_{0}^{\infty} g_{2}^{1-\tau}(s) d s \leq \int_{0}^{\infty} \frac{1}{\left[(\sigma-1)\left(\int_{t_{0}}^{t} \xi(s) d s\right)+g_{2}\left(t_{0}\right)^{1-\sigma}\right]^{(1-\tau) /(\sigma-1)}} d s
\end{aligned}
$$

For $0<\tau<2-\sigma<1$, we have $(1-\tau) /(\sigma-1)>1$ and (15), we find

$$
\begin{aligned}
& \int_{0}^{\infty} g_{1}^{1-\tau}(s) d s<\infty, \quad \forall 0<\tau<2-\sigma, \\
& \int_{0}^{\infty} g_{2}^{1-\tau}(s) d s<\infty, \quad \forall 0<\tau<2-\sigma .
\end{aligned}
$$

From (72) (for $\theta=\tau$ and $\rho=\sigma$ ) and (55) gives

$$
\begin{aligned}
\left(g_{1} \circ u_{x}\right)(t) \leq & C_{1}\left(E(0) \int_{0}^{\infty} g_{1}^{1-\tau}(s) d s\right)^{(\sigma-1) /(\sigma-1+\tau)} \\
& \cdot\left(\left(g_{1}^{\sigma} \circ u_{x}\right)(t)\right)^{\tau /(\sigma-1+\tau)} \\
\leq & C_{1}^{\prime}\left(\left(g_{1}^{\sigma} \circ v_{x}\right)(t)\right)^{\tau /(\sigma-1+\tau)}
\end{aligned}
$$

Similarly, we have

$$
\left(g_{2} \circ v_{x}\right)(t) \leq C_{2}^{\prime}\left(\left(g_{2}^{\sigma} \circ v_{x}\right)(t)\right)^{\tau /(\sigma-1+\tau)} \text {, }
$$


for some $C_{1}^{\prime}, C_{2}^{\prime}>0$. Hence, $\forall \sigma_{1}>1$, we find

$$
\begin{aligned}
E^{\sigma_{1}}(t) \leq & C^{\prime \prime} E^{\sigma_{1}-1}(0)\left(\int_{0}^{L} x u_{t}^{2} d x+\int_{0}^{L} x v_{t}^{2} d x+\int_{0}^{L} x u_{x}^{2} d x\right. \\
& \left.+\int_{0}^{L} x v_{x}^{2} d x-\int_{0}^{L} x\left[a|u+v|^{2(r+2)}+2 b|u v|^{r+2}\right] d x+K(z, y)\right) \\
& +C_{1}^{\prime}\left(\left(g_{1} \circ u_{x}\right)(t)\right)^{\sigma_{1}}+C_{2}^{\prime}\left(\left(g_{2} \circ v_{x}\right)(t)\right)^{\sigma_{1}} \\
\leq & C^{\prime \prime} E^{\sigma_{1}-1}(0)\left(\int_{0}^{L} x u_{t}^{2} d x+\int_{0}^{L} x v_{t}^{2} d x+\int_{0}^{L} x u_{x}^{2} d x\right. \\
& \left.+\int_{0}^{L} x v_{x}^{2} d x-\int_{0}^{L} x\left[a|u+v|^{2(r+2)}+2 b|u v|^{r+2}\right] d x+K(z, y)\right) \\
& +C_{1}^{\prime}\left(\left(g_{1}^{\sigma} \circ u_{x}\right)(t)\right)^{\tau \sigma_{1} /(\sigma-1+\tau)}+C_{2}^{\prime}\left(\left(g_{2}^{\sigma} \circ v_{x}\right)(t)\right)^{\tau \sigma_{1} /(\sigma-1+\tau)}
\end{aligned}
$$

We choose $\tau=1 / 2$ and $\sigma_{1}=2 \sigma-1$ (therefore, $\tau \sigma_{1} /(\sigma-1+\tau)=1$ ) and (144); we get, for some $\Gamma>0$,

$$
\begin{aligned}
E^{\sigma_{1}}(t) \leq & \Gamma\left[\int_{0}^{L} x u_{t}^{2} d x+\int_{0}^{L} x v_{t}^{2} d x+\int_{0}^{L} x u_{x}^{2} d x+\int_{0}^{L} x v_{x}^{2} d x\right. \\
& +K(z, y)-\int_{0}^{L} x\left[a|u+v|^{2(r+2)}+2 b|u v|^{r+2}\right] d x \\
& \left.+\left(g_{1}^{\sigma} \circ u_{x}\right)(t)+\left(g_{2}^{\sigma} \circ v_{x}\right)(t)\right] .
\end{aligned}
$$

By combining (72), (137), and (151), we find

$F^{\prime}(t) \leq-\frac{\sigma}{\Gamma} \xi(t) E^{\sigma_{1}}(t) \leq-\frac{\sigma}{\Gamma} \alpha_{1}^{\sigma_{1}} F^{\sigma_{1}}(t) \xi(t), \forall t \geq t_{0}$.

By integrating (153) gives

$$
F(t) \leq C_{1}^{*}\left(1+\int_{t_{0}}^{t} \xi(s) d s\right)^{-\left(1 /\left(\sigma_{1}-1\right)\right)}, \quad \forall t \geq t_{0} .
$$

Hence,

$$
\int_{t_{0}}^{\infty} F(t) d t \leq C_{1}^{*} \int_{t_{0}}^{\infty} \frac{1}{\left(1+\int_{t_{0}}^{t} \xi(s) d s\right)^{1 /\left(\sigma_{1}-1\right)}} d t
$$

From $\left(1 /\left(\sigma_{1}-1\right)\right)>0$ and $\left(1+\int_{t_{0}}^{t} \xi(s) d s\right) \longrightarrow+\infty$ as $t \longrightarrow+\infty$, we find

$$
\int_{t_{0}}^{\infty} F(t) d t<\infty
$$

Also, we use (24), and we get

$$
t F(t) \leq \frac{C_{1}^{*} t}{\left(1+\int_{t_{0}}^{t} \xi(s) d s\right)^{1 /\left(\sigma_{1}-1\right)}} \leq C_{\sigma}
$$

Hence, we find

$$
\sup _{t \geq t_{0}} t F(t)<\infty
$$

From $E(t)$ which is bounded, using (72), (156), and (158) to get

$$
\int_{t_{0}}^{\infty} F(t) d t+\sup _{t \geq 0}(t F(t))<\infty
$$

Therefore, using (55) and Lemma $10($ for $\rho=\sigma)$ gives

$$
\begin{aligned}
\left(g_{1} \circ u_{x}\right)(t) \leq & C_{2}^{*}\left(t\left\|u_{x}(x, t)\right\|_{H}^{2}+\int_{0}^{t}\left\|u_{x}(x, s)\right\|_{H}^{2} d s\right)^{(\sigma-1) / \sigma} \\
& \times\left(\int_{0}^{t} g^{\sigma}(t-s)\left\|u_{x}(x, t)-u_{x}(x, s)\right\|_{H}^{2} d s\right) \\
\leq & C_{2}^{*}\left(t F(t)+\int_{t_{0}}^{t} F(s) d s\right)^{(\sigma-1) / \sigma}\left(\left(g_{1}^{\sigma} \circ u_{x}\right)(t)\right)^{1 / \sigma} \\
\leq & C_{3}^{*}\left(\left(g_{1}^{\sigma} \circ u_{x}\right)(t)\right)^{1 / \sigma} .
\end{aligned}
$$

This means

$$
\begin{aligned}
& \left(g_{1}^{\sigma} \circ u_{x}\right)(t) \geq C_{4}\left(\left(g_{1} \circ u_{x}\right)(t)\right)^{\sigma}, \\
& \left(g_{2}^{\sigma} \circ v_{x}\right)(t) \geq C_{5}\left(\left(g_{2} \circ v_{x}\right)(t)\right)^{\sigma},
\end{aligned}
$$

for some $C_{4}, C_{5}>0$.

Then, combining (137), (161), and (162) yields

$$
\begin{aligned}
F^{\prime}(t) \leq & -C_{6} \xi(t)\left\{\int_{0}^{L} x u_{t}^{2} d x+\int_{0}^{L} x v_{t}^{2} d x+\int_{0}^{L} x u_{x}^{2} d x\right. \\
& +\int_{0}^{L} x v_{x}^{2} d x-\int_{0}^{L} x\left[a|u+v|^{2(r+2)}+2 b|u v|^{r+2}\right] d x \\
& \left.+K(z, y)+\left(\left(g_{1} \circ u_{x}\right)(t)\right)^{\sigma}+\left(\left(g_{2} \circ v_{x}\right)(t)\right)^{\sigma}\right\}
\end{aligned}
$$

for some $C_{6}>0$.

As in [1], we obtain

$$
\begin{aligned}
E^{\sigma}(t) \leq & C_{7} \xi(t)\left\{\int_{0}^{L} x u_{t}^{2} d x+\int_{0}^{L} x v_{t}^{2} d x+\int_{0}^{L} x u_{x}^{2} d x\right. \\
& +\int_{0}^{L} x v_{x}^{2} d x-\int_{0}^{L} x\left[a|u+v|^{2(r+2)}+2 b|u v|^{r+2}\right] d x \\
& \left.+K(z, y)+\left(\left(g_{1} \circ u_{x} t\right)(t)\right)^{\sigma}+\left(\left(g_{2} \circ v_{x}\right)(t)\right)^{\sigma}\right\}
\end{aligned}
$$

$\forall t \geq 0$ and some $C_{7}>0$. 
Combining (163), (164), and (72), we find

$$
F^{\prime}(t) \leq-C_{8} \xi(t) F^{\sigma}(t), \forall t \geq t_{0},
$$

for some $C_{8}>0$.

By integrating (163) over $\left(t_{0}, t\right)$, we get

$$
F(t) \leq C_{9}\left(1+\int_{t_{0}}^{t} \xi(s) d s\right)^{-(1 /(\sigma-1))}, \quad \forall t \geq t_{0}
$$

Hence, $(129)_{2}$ is showed by (72) as well.

\section{Data Availability}

No data were used to support this study.

\section{Conflicts of Interest}

This work does not have any conflicts of interest.

\section{Acknowledgments}

The researchers would like to thank the Deanship of Scientific Research, Qassim University, for funding the publication of this project.

\section{References}

[1] B. Cahlon, D. M. Kulkarni, and P. Shi, "Stepwise stability for the heat equation with a nonlocal constraint," SIAM Journal on Numerical Analysis, vol. 32, no. 2, pp. 571-593, 1995.

[2] J. R. Cannon, "The solution of the heat equation subject to the specification of energy," Quarterly of Applied Mathematics, vol. 21, no. 2, pp. 155-160, 1963.

[3] V. Capasso and K. Kunisch, "A reaction-diffusion system arising in modelling man-environment diseases," Quarterly of Applied Mathematics, vol. 46, no. 3, pp. 431-450, 1988.

[4] Y.S. Choi and K. Y. Chan, "A parabolic equation with nonlocal boundary conditions arising from electrochemistry," Nonlinear Analysis: Theory, Methods \& Applications, vol. 18, no. 4, pp. 317-331, 1992.

[5] A. Choucha, D. Ouchenane, K. Zennir, and F. Feng, "Global well-posedness and exponential stability results of a class of Bresse-Timoshenko-type systems with distributed delay term," Mathematical Methods in the Applied Sciences, vol. 42, pp. 1-26, 2020.

[6] C. Mu and J. Ma, "On a system of nonlinear wave equations with Balakrishnan-Taylor damping," Zeitschrift für Angewandte Mathematik und Physik, vol. 65, no. 1, pp. 91-113, 2014.

[7] R. E. Ewing and T. Lin, "A class of parameter estimation techniques for fluid flow in porous media," Advances in Water Resources, vol. 14, no. 2, pp. 89-97, 1991.

[8] M. A. Goodrich and M. A. Ragusa, "Hölder continuity of weak solutions of p-Laplacian PDEs with VMO coefficients," Nonlinear Analysis-Theory Methods and Applications, vol. 185, pp. 336-355, 2019.

[9] J. Hao and F. Wang, "Energy decay in a Timoshenko-type system for thermoelasticity of type III with distributed delay and past history," Electronic Journal of Differential Equations, vol. 2018, pp. 1-27, 2018.

[10] E. Pişkin and F. Ekinci, "General decay and blowup of solutions for coupled viscoelastic equation of Kirchhoff type with degenerate damping terms," Mathematical Methods in the Applied Sciences, vol. 46, pp. 5468-5488, 2019.

[11] N. I. Ionkin and E. I. Moiseev, "A problem for the heat conduction equation with two-point boundary condition," Differentsial'nye Uravneniya, vol. 15, pp. 1284-1295, 1979.

[12] N. I. Ionkin, "Solution of boundary value problem in heat conduction theory with nonclassical boundary conditions," Differentsial'nye Uravneniya, vol. 13, pp. 1177-1182, 1977.

[13] A. V. Kartynnik, "Three-point boundary value problem with an integral space-variable condition for a second order parabolic equation," Differential Equations, vol. 26, pp. 11601162, 1990.

[14] L. I. Kamynin, "A boundary-value problem in the theory of heat conduction with non-classical boundary conditions," USSR Computational Mathematics and Mathematical Physics, vol. 4, pp. 1006-1024, 1964.

[15] M. R. Li and L. Y. Tsai, "Existence and nonexistence of global solutions of some system of semilinear wave equations," Nonlinear Analysis: Theory, Methods \& Applications, vol. 54, no. 8, pp. 1397-1415, 2003.

[16] L. S. Pulkina, "A nonlocal problem with integral conditions for hyperbolic equations," Electronic Journal of Differential Equations, vol. 45, pp. 1-6, 1999.

[17] L. S. Pul'kina, "TheL ${ }^{2}$ solvability of a nonlocal problem with integral conditions for a hyperbolic equation," Differential Equations, vol. 36, no. 2, pp. 316-318, 2000.

[18] A. S. Nicaise and C. Pignotti, "Stabilization of the wave equation with boundary or internal distributed delay," Differential and Integral Equations, vol. 21, no. 9-10, pp. 935-958, 2008.

[19] M. A. Ragusa and A. Tachikawa, "Partial regularity of the minimizers of quadratic functionals with VMO coefficients," Journal of the London Mathematical Society, vol. 72, no. 3, pp. 609-620, 2005.

[20] M. A. Ragusa and A. Tachikawa, "Regularity for minimizers for functionals of double phase with variable exponents," Adv. Nonlinear Anal., vol. 9, no. 1, pp. 710-728, 2019.

[21] P. Shi and M. Shillor, "On design of contact patterns in one dimensional thermoelasticity," in Theoretical Aspects of Industrial Design, SIAM, Philadelphia, PA, USA, 1992.

[22] P. Shi, "Weak solution to an evolution problem with a nonlocal constraint," SIAM Journal on Mathematical Analysis, vol. 24, no. 1, pp. 46-58, 1993.

[23] W. Shuntang, "Blow-up of solutions for a singular nonlocal viscoelastic equation," Journal of Partial Differential Equations, vol. 24, pp. 140-149, 2018.

[24] N. I. Yurchuk, "Mixed problem with an integral condition for certain parabolic equations," Differentsial'nye Uravneniya, vol. 22, pp. 2117-2126, 1986.

[25] S. Boulaaras, R. Guefaifia, and N. Mezouar, "Global existence and decay for a system of two singular one-dimensional nonlinear viscoelastic equations with general source terms," Applicable Analysis, pp. 1-25, 2020.

[26] S. Boulaaras and N. Mezouar, "Global existence and decay of solutions of a singular nonlocal viscoelastic system with a nonlinear source term, nonlocal boundary condition, and localized damping term," Mathematical Methods in the Applied Sciences, vol. 43, no. 10, pp. 6140-6164, 2020. 
[27] T. A. Apalara, "Uniform decay in weakly dissipative Timoshenko system with internal distributed delay feedbacks," Acta Mathematica Scientia, vol. 36, no. 3, pp. 815-830, 2016.

[28] S. Boulaaras, A. Choucha, D. Ouchenane, and B. Cherif, "Blow up of solutions of two singular nonlinear viscoelastic equations with general source and localized frictional damping terms," Adv. Difference Equ., vol. 2020, no. 1, article 310, 2020.

[29] W. Liu, Y. Sun, and G. Li, "On decay and blow-up of solutions for a singular nonlocal viscoelastic problem with a nonlinear source term," Topological Methods in Nonlinear Analysis, vol. 49, pp. 299-323, 2017.

[30] A. Draifia, A. Zarai, and S. Boulaaras, "Global existence and decay of solutions of a singular nonlocal viscoelastic system," Rendiconti del Circolo Matematico di Palermo Series 2, vol. 69, no. 1, pp. 125-149, 2020.

[31] N. Mezouar and S. Boulaaras, "Global existence and decay of solutions of a singular nonlocal viscoelastic system with damping terms," Topological Methods in Nonlinear Analysis, vol. 56, no. 1, pp. 283-312, 2020. 